\title{
Characterization of Fungi Associated With Wood Decay of Tree Species and Grapevine in Greece
}

Emmanouil A. Markakis ${ }^{\dagger}$ and Nektarios Kavroulakis, Laboratory of Plant Pathology, Institute of Olive Tree, Subtropical Crops and Viticulture, Hellenic Agricultural Organization Demeter (HAO-Demeter), Agrokipio 73100, Chania, Crete, Greece; Spyridon Ntougias, Laboratory of Wastewater Management and Treatment Technologies, Department of Environmental Engineering, Democritus University of Thrace, 67100, Xanthi, Greece; Georgios C. Koubouris and Chrysi K. Sergentani, Laboratory of Olive Cultivation, Institute of Olive Tree, Subtropical Crops and Viticulture, HAO-Demeter, Agrokipio 73100, Chania, Crete, Greece; and Eleftherios K. Ligoxigakis, Laboratory of Plant Pathology, Department of Viticulture, Vegetable Crops, Floriculture and Plant Protection, HAO-Demeter, Mesa Katsampas 71003, Iraklio, Crete, Greece

\begin{abstract}
A two-year survey was conducted to identify fungi associated with wood decay in a range of tree species and grapevine. Fifty-eight fungal strains isolated from plants of 18 species showing typical wood decay symptoms were characterized by morphological, physiological, and molecular analyses. By $5.8 \mathrm{~S}$ rRNA gene-ITS sequencing analysis, these isolates were classified into 25 distinct operational taxonomic units, including important phytopathogenic species of the phyla Pezizomycotina and Agaricomycotina, such as Fomitiporia, Inonotus, Phellinus, Inocutis, Fuscoporia, Trametes, Fusarium, Eutypa, Phaeomoniella, Phaeoacremonium, and Pleurostomophora spp. The white rot basidiomycetes Fomitiporia mediterranea (20 isolates, 34.5\%) and Inonotus hispidus (6 isolates, 10.3\%) were the most prevalent. Pathogenicity tests revealed for the first time that certain fungal species of the genera Fomitiporia,

Inonotus, Phellinus, Pleurostomophora, and Fusarium caused wood infection of various tree species in Greece and worldwide. To the best of our knowledge, this is the first report of $F$. mediterranea as the causal agent of wood decay in pear, pomegranate, kumquat, and silk tree. This is also the first record of Inonotus hispidus, Phellinus pomaceus, Pleurostomophora richardsiae, and Fusarium solani in apple, almond, avocado, and mulberry tree, respectively, whereas $P$. richardsiae was associated with wood infection of olive tree for the first time in Greece. Cross pathogenicity tests with $F$. mediterranea strains originated from grapevine applied on other woody hosts and from olive on grapevine demonstrated partial host specificity of the fungus. The potential of $F$. mediterranea to transinfect hosts other than those originated, along with the host range extension of the fungus, is discussed.
\end{abstract}

Wood decay diseases are caused by a wide range of fungi, affecting several tree species and grapevine worldwide (Fischer 2006; Roccotelli et al. 2014). Most wood-degrading microorganisms are white- or brownrot basidiomycetes that can decompose the lignified cell walls of the woody plants (Blanchette 1991). These fungi can cause decay and discoloration of wood tissues, in particular in the trunk, branches, and roots. Although only a few of these fungi are directly responsible for tree mortality, wood-rot basidiomycetes lead to structural deterioration of woody tissues and economic damage due to the premature aging, decline, and substantially reduced crop productivity (Butin 1995; Lonsdale 1999). Apart from wood decay Basidiomycota, other fungi like Fusarium, Phaeoacremonium, Phaeomoniella, and Pleurostomophora strains have been reported as wood-penetrating agents of several plant species (Carlucci et al. 2013; Graham et al. 1985; Mugnai et al. 1999). Most of them penetrate through wounds, although several wood decaying species can invade the uninjured surface directly (Roccotelli et al. 2014).

A well-known basidiomycete associated with wood decay of grapevine (Vitis vinifera L.) in European grapevine-growing countries is Fomitiporia mediterranea M. Fisch. (Fischer 2002). This fungus has also been reported as the causal agent of heart wood rot in kiwifruit (Actinidia deliciosa L.) (Di Marco et al. 2004; Elena and Paplomatas 2002), olive (Olea europaea L.) (Fischer 2006; Paplomatas et al. 2006a, b), orange (Citrus sinensis L.), lemon (C. limon L.), mandarin $(C$. reticulate $\mathrm{L}$.), and sour orange (C. aurantium L.) trees (Elena et al. 2006; Roccotelli et al. 2014). In addition, $F$. mediterranea has been detected in plane trees (Platanus $\times$ acerifolia) affected by canker diseases (Pilotti et al. 2005) and hazelnut trees (Corylus avellana L.) showing decay and decline (Pilotti et al. 2010). Moreover, the same species has been found on boxelder (Acer negundo L.), crape myrtle (Lagestroemia indica L.), bay tree (Laurus

${ }^{\dagger}$ Corresponding author. E-mail: markmanos1979@yahoo.gr

Accepted for publication 25 June 2017

C 2017 The American Phytopathological Society nobilis L.), common privet (Ligustrum vulgare L.), evergreen oak (Quercus ilex L.), and black locust (Robinia pseudoacacia L.) (Fischer 2006).

Pathogenicity tests revealed that citrus-originated $F$. mediterranea isolates were pathogenic on this particular host while isolates of this fungus from grapevine and kiwi were nonpathogenic to citrus trees (Elena et al. 2006; Roccotelli et al. 2014), suggesting either host specificity or involvement of host-specific cryptic species (Pilotti et al. 2010). On the other hand, $F$. mediterranea is not limited to grapevines, but also occurs in other tree species, indicating the potential role of other hosts (except grapevine) to serve as inoculum sources for the fungus to infect grapevines (Surico et al. 2006; 2008). However, the hypothesis that $F$. mediterranea isolates from non-Vitis hosts are pathogenic on grapevine has not been verified yet (Surico et al. 2006).

In the last decades, several tree species and grapevine with typical wood decay symptoms have been brought to phytopathological laboratories, demanding accurate disease diagnosis. Besides grapevine, olive, citrus, apple (Malus domestica L.), and pomegranate (Punica granatum $\mathrm{L}$.) trees are plant species with high decline and/or dieback disease incidence, followed by other cultivated and ornamental species that are occasionally observed to exhibit such decay. For instance, the extensive damage of olive trees in Messinia and Arkadia prefectures in Greece and elsewhere, caused by $F$. mediterranea and various species primarily in the Botryosphaeriaceae, has been previously reported (Carlucci et al. 2013; Paplomatas et al. 2006a, b; ÚrbezTorres et al. 2013). Recently, serious damage in olive orchards has also been observed in Evros prefecture and in Crete, southern Greece (present study). Moreover, citrus wood rot is commonly found in old citrus trees throughout the globe and is due to the infection caused by endemic wood decay species (Bigelow et al. 1996; 1998; Elena et al. 2006; Mayorquin et al. 2016; Roccotelli et al. 2014). However, in most cases, the causal agents of these wood-decay-associated diseases remained unidentified. In addition, the reason for the significant increase of the disease incidence in woody hosts other than grapevine has not been extensively investigated.

Although wood decay fungi are considered as important primary decomposers in forest habitats, they can also lead to economic damage 
in cultivated fields, owing to tree decline and death (Butin 1995; Elena et al. 2006; Lonsdale 1999). Therefore, the main objectives of this work were: (i) to characterize fungi associated with wood decay of tree species and grapevine in Greece; (ii) to test the potential of $F$. mediterranea originated from grapevine to transinfect other hosts; and (iii) to investigate whether $F$. mediterranea from other plant species is also capable of infecting grapevine.

\section{Materials and Methods}

Sampling. Wood samples with typical decay symptoms as well as fruiting bodies of potential wood-decay fungi were collected over a 2-year period (2013-2014), during field surveys in orchards and boulevards located mostly in Crete (southern Greece). Some samples from olive orchards in Peloponnese (south-central Greece) and Thrace (northeastern Greece) were also collected and analyzed (Table 1). Woody samples were mainly received during the pruning period (November to February) and consisted of pieces of wood with decay symptoms from trunks and large branches of diseased trees.

Isolation of fungi. To obtain the potential causal agents of wood decay, samples were cut crosswise and lengthwise and wood fragments $(\sim 10 \mathrm{~cm}$ in length) were surface-disinfected by $95 \%$ ethyl alcohol for $2 \mathrm{~min}$. For each sample, 10 xylem chips from the center of the white- or brown-colored spongy area and 10 additional chips from the surrounding brown, hard, necrotic wood zone were aseptically placed onto acidified potato dextrose agar (PDA). In the case of fruiting bodies, tissues were surface-disinfected by $95 \%$ ethyl alcohol and small pieces of internal tissues were transferred onto acidified PDA. Plates were incubated at $24^{\circ} \mathrm{C}$ in the dark for 3 weeks. The emerging fungal colonies that grew out of tissue excisions were examined visually and under a light microscope. The fungi isolated consistently and steadily from each sample were transferred onto new PDA plates. For short-term storage, all fungal isolates were maintained on PDA at $4{ }^{\circ} \mathrm{C}$.

Morphological and cultural characterization of isolates. Mycelial growth of the wood decay strains isolated was examined by transferring PDA agar plugs of actively growing mycelium ( $7 \mathrm{~mm}$ in diameter) into new PDA plates. Plates (three per isolate) were incubated at $24^{\circ} \mathrm{C}$ in the dark and the colonies' diameter was measured at day $3,7,10,14,17$, and 21 or stopped earlier, when the plate surface was completely covered. The growth rate of fungal isolates was expressed in $\mathrm{mm} /$ day. At the end of the incubation period, colony characteristics (color, mycelium, colony type, and shape) were observed. Available conidia and hyphal features (color, shape, presence, or absence of septum and clamp) were also recorded. Pigmentation of $F$. mediterranea isolates was visually assessed by recording the appearance of pigment on the underside and upper side of PDA plates. All F. mediterranea cultures were scored in a five-point evaluation scale regarding the percentage of pigmented area with reference to the entire culture surface, where: $0=$ white colony; $1=1$ to $25 \% ; 2=26$ to $50 \% ; 3=51$ to $75 \%$; and $4=76$ to $100 \%$ of culture surface pigmented. Isolates with an average pigment grade of $\geq 2.0$ on the underside of plates were classified into type $S$ (staining of the culture medium), whereas the rest were fallen into type B (bleaching of the culture medium) (Fischer 2006). Moreover, mycelium abundance of $F$. mediterranea isolates was based on a visual scale from 0 to 4, where: $0=$ yeast-like appearance; $1=$ sparse development of aerial hyphae; 2 = cottony mycelial culture; 3 = modest woolly appearance; and $4=$ intensive woolly mycelium. To evaluate and describe the microscopic features of the colonies, actively growing mycelia from culture's periphery were prepared and microscopic observations (30 readings per isolate) were carried out.

Sequencing of internal transcribed spacer regions. To molecularly characterize fungal isolates, the internal transcribed spacer (ITS) regions of ribosomal RNA gene were sequenced. Fungal DNA was extracted from 150 to $200 \mathrm{mg}$ mycelium (fresh weight) by using the NucleoSpin Plant II genomic DNA extraction kit (MachereyNagel, Düren, Germany) and amplified with the use of universal primers ITS1 and ITS4 (White et al. 1990). All PCR assays were carried out in an MJ Research PTC-200 Gradient thermocycler, by the use of the KAPA Taq ReadyMix PCR kit (Kapa Biosystems, Wilmington, MA, USA). PCR performance included denaturation at $94^{\circ} \mathrm{C}$ for $3 \mathrm{~min}$; followed by 34 cycles of 1 min of denaturation at $94^{\circ} \mathrm{C}, 40 \mathrm{~s}$ of annealing at $54^{\circ} \mathrm{C}$, and $40 \mathrm{~s}$ of extension at $72^{\circ} \mathrm{C}$; and a final extension step at $72^{\circ} \mathrm{C}$ for $15 \mathrm{~min}$. Amplified products were purified with the NucleoSpin Gel and PCR Clean-up kit (Macherey-Nagel) and sequenced by both forward and reverse primers at Macrogen Europe (Amsterdam). The CAP3 Sequence Assembly Program was used to edit the raw sequencing data (Huang and Madan 1999) and the sequences assembled were deposited in GenBank as Accession Nos. KX881579 to KX881637. The latter were aligned by the Clustal Omega platform (Sievers et al. 2011) and estimation of sequence relatedness was calculated by the Tamura-Nei maximum-likelihood algorithm (Tamura and Nei 1993). Trees construction was carried out by MEGA (Tamura et al. 2013) through topology estimation by the neighbor-joining method (Saitou and Nei 1987) and bootstrap analysis of 1000 trees.

Identification and characterization of isolates. Morphological and physiological features along with ITS sequences were used to characterize the fungal isolates obtained from the diseased tissues. Molecular identification of the wood decay isolates was carried out using the BLASTn option at NCBI and comparing their ITS sequences with those from GenBank database. Fomitiporia mediterranea strain K.E. Fomit4, belonging to Benaki Phytopathological Institute Collection (BPIC), was provided by Benaki Phytopathological Institute, Kifissia, Athens, Greece, and served as the reference strain.

Pathogenicity and cross-pathogenicity tests. In order to confirm pathogenicity, $F$. mediterranea isolates A2LKP1, E3LK1, R1LK1, K1KP1, AXL1LK1, and AKKON1LK1 originated from grapevine, olive, pomegranate, kumquat (Fortunella japonica Swingle), pear (Pyrus communis L.), and silk tree (Albizia julibrissin Durazz), respectively, were used to inoculate healthy plants of the same species. In addition, the pathogenicity of Pleurostomophora richardsiae (Nannf.) L. Mostert, W. Gams \& Crous isolates E1PK1 and AV1LK3 originated from olive and avocado (Persea americana Mill.), respectively, was tested on healthy plants of the same species. Moreover, the isolates A2LP1 (Phaeomoniella chlamydospora W. Gams, Crous, M.J. Wingf. \& Mugnai), MOUR2P3 (Fusarium solani (Mart.) Sacc.), AM2XL1 (Phellinus pomaceus (Pers.) Maire), and MP1 (Inonotus hispidus (Bull.) P. Karst.) originated from grapevine, mulberry (Morus platanifolia L.), almond (Prunus dulcis Mill.), and apple tree, respectively, were used to inoculate healthy plants of the same species. Furthermore, cross-pathogenicity tests were conducted to evaluate the pathogenicity of $F$. mediterranea isolate A2LKP1 from grapevine on olive, pomegranate, kumquat, almond, and apple trees, as well as to test whether $F$. mediterranea isolate E3LK1 from olive is also pathogenic on grapevine.

Nine 2-year-old trees of each plant species (except the silk tree) were inoculated on April 2014. Artificial inoculation was performed by making a $5.5-\mathrm{mm} \times 10.0-\mathrm{mm}$ hole in the trunk through the use of a Black \& Decker EPC 12 drilling apparatus, inserting one 5.5-mmdiameter mycelial disc taken from the margin of a 15-day-old PDA culture, sealing the hole with vaseline and fastening the area with paper tape to protect the inoculum. Nine additional trees of each plant species were mock-inoculated with sterilized PDA and served as controls. Trees were placed under ambient conditions, in an adjacent experimental field of Institute of the Olive Tree, Subtropical Crops, and Viticulture in Chania, Crete, Greece. Each treatment consisted of three trees and the experiment was replicated three times (nine trees per treatment in total).

In April 2015, nine 8-month-old silk tree plants were artificially inoculated with $F$. mediterranea isolate AKKON1LK1 originated from a 20-year-old silk tree, as follows: two 5.5-mm-diameter mycelial discs taken from the margin of a 15-day-old PDA culture were placed in a 1.5-ml Eppendorf tube and the cover was removed; then, the apical end of the stem was cut with sterilized pruning scissors and the tube was adjusted onto stem, so as the fresh pruning wound to be in touch with the mycelial discs. Nine additional plants were mockinoculated with sterilized PDA and served as controls. The tube together with the stem were fastened with cellophane membrane and covered with adhesive paper tape to protect the inoculum. At the same time, the abovementioned technique was also used to test the pathogenicity of $F$. mediterranea isolate E3LK1 from olive on nine 
1-year-old grapevine plants. Due to the low extension of wood discoloration in pomegranate and kumquat trees inoculated by employing the trunk-drilling technique, 10 branches with a diameter of 6 to $8 \mathrm{~mm}$ from each pomegranate, kumquat, and olive tree were artificially inoculated with $F$. mediterranea isolates R1LK1, K1KP1, and E3LK1, respectively, to verify the efficiency of the pruning- wound inoculation method. Branches mock-inoculated with sterilized PDA discs served as controls.

Disease assessment. Trunk-drilled inoculated trees were cut above soil level in November 2014 (7-months post inoculation) and longitudinal and transverse sections of trunks were performed to measure the extension of wood tissue symptoms above and below

Table 1. Morphological and physiological features of the fungal isolates (mean \pm SE) analyzed in the present study, along with their host, geographic origin, and isolation year

\begin{tabular}{|c|c|c|c|c|c|c|c|c|}
\hline Isolate $^{w}$ & Host & Substrate $^{\mathrm{x}}$ & Location & Year & $\begin{array}{c}\text { Growth } \\
\text { rate }(\mathrm{mm} / \text { day })^{y}\end{array}$ & Colony color & $\begin{array}{c}\text { Conidial } \\
\text { dimensions }(\mu \mathrm{m})^{\mathbf{z}}\end{array}$ & $\begin{array}{c}\text { Hyphae } \\
\text { width }(\mu \mathrm{m})\end{array}$ \\
\hline E1KP1 & Olive tree & Wood & Nileas, Kalamata & 2013 & $3.92 \pm 0.05$ & yellow to pale brown & $\mathrm{nf}$ & $2.43 \pm 0.11$ \\
\hline E1PK1 & Olive tree & Wood & Nileas, Kalamata & 2013 & $1.81 \pm 0.16$ & light to dark brown & $4.79( \pm 0.25) \times 2.41( \pm 0.07)$ & $1.98 \pm 0.10$ \\
\hline E2mXL1 & Olive tree & Fruiting body & Kissamos, Chania & 2014 & $4.51 \pm 0.07$ & white to pale yellow & $\mathrm{nf}$ & $2.67 \pm 0.22$ \\
\hline E2LK3 & Olive tree & Wood & Kissamos, Chania & 2014 & $4.22 \pm 0.16$ & white to pale yellow & $\mathrm{nf}$ & $2.76 \pm 0.21$ \\
\hline E3LK1 & Olive tree & Wood & Kissamos, Chania & 2014 & $3.44 \pm 0.05$ & white to yellow & $\mathrm{nf}$ & $2.54 \pm 0.13$ \\
\hline E5LK1 & Olive tree & Fruiting body & Platanias, Chania & 2014 & $1.89 \pm 0.13$ & pale brown to golden & $\mathrm{nf}$ & $2.85 \pm 0.10$ \\
\hline E6LK1 & Olive tree & Wood & Apokoronas, Chania & 2014 & $4.39 \pm 0.04$ & white to golden & $\mathrm{nf}$ & $2.56 \pm 0.12$ \\
\hline E7LK1 & Olive tree & Wood & Apokoronas, Chania & 2014 & $4.39 \pm 0.04$ & white to pale yellow & $\mathrm{nf}$ & $2.72 \pm 0.16$ \\
\hline E9mLK1 & Olive tree & Fruiting body & Makri, Alexandroupolis & 2014 & $6.23 \pm 0.16$ & pale brown & $\mathrm{nf}$ & $2.47 \pm 0.14$ \\
\hline E10mLK1 & Olive tree & Fruiting body & Makri, Alexandroupolis & 2014 & $4.29 \pm 0.03$ & white to pale golden & $\mathrm{nf}$ & $2.68 \pm 0.14$ \\
\hline E11mLK1 & Olive tree & Fruiting body & Makri, Alexandroupolis & 2014 & $3.10 \pm 0.05$ & white to golden & $\mathrm{nf}$ & $2.35 \pm 0.08$ \\
\hline A2LKP1 & Grapevine & Wood & Episkopi, Iraklio & 2014 & $3.41 \pm 0.11$ & white to golden & $\mathrm{nf}$ & $2.78 \pm 0.15$ \\
\hline A2LP1 & Grapevine & Wood & Episkopi, Iraklio & 2014 & $1.14 \pm 0.03$ & olive-green & $4.53( \pm 0.20) \times 1.88( \pm 0.08)$ & $2.64 \pm 0.11$ \\
\hline A3LKP2 & Grapevine & Wood & Agios Thomas, Iraklio & 2014 & $4.60 \pm 0.17$ & white to pale golden & $\mathrm{nf}$ & $2.84 \pm 0.19$ \\
\hline A4LKP1 & Grapevine & Wood & Garipa, Iraklio & 2014 & $4.08 \pm 0.13$ & white to golden & $\mathrm{nf}$ & $2.75 \pm 0.20$ \\
\hline A5LP1 & Grapevine & Wood & Kolimpari, Chania & 2014 & $1.24 \pm 0.01$ & dark olive-green & $4.36( \pm 0.20) \times 2.04( \pm 0.08)$ & $2.71 \pm 0.10$ \\
\hline A5LKK1 & Grapevine & Wood & Kolimpari, Chania & 2014 & $0.84 \pm 0.04$ & pale orange & $5.50( \pm 0.25) \times 2.59( \pm 0.13)$ & $2.65 \pm 0.09$ \\
\hline A5LV1 & Grapevine & Wood & Kolimpari, Chania & 2014 & $3.50 \pm 0.08$ & white to pale olive-green & $\mathrm{nf}$ & $2.70 \pm 0.13$ \\
\hline P1KP1 & Orange tree & Wood & Platanias, Chania & 2014 & $4.67 \pm 0.08$ & white to pale golden & $\mathrm{nf}$ & $3.03 \pm 0.20$ \\
\hline P2KP1 & Orange tree & Wood & Apokoronas, Chania & 2014 & $4.38 \pm 0.05$ & white to pale golden & $\mathrm{nf}$ & $2.75 \pm 0.12$ \\
\hline R1LK1 & Pomegranate tree & Wood & Agios Thomas, Iraklio & 2014 & $3.82 \pm 0.14$ & white to pale golden & nf & $2.63 \pm 0.19$ \\
\hline R1K1 & Pomegranate tree & Wood & Agios Thomas, Iraklio & 2014 & $3.25 \pm 0.04$ & pale yellow & $6.32( \pm 0.30) \times 2.24( \pm 0.09)$ & $2.49 \pm 0.09$ \\
\hline R1LKP1 & Pomegranate tree & Wood & Agios Thomas, Iraklio & 2014 & $3.95 \pm 0.03$ & white to pale golden & $\mathrm{nf}$ & $2.28 \pm 0.10$ \\
\hline R1YKL1 & Pomegranate tree & Wood & Agios Thomas, Iraklio & 2014 & $3.63 \pm 0.13$ & beige to olive-green & $9.23( \pm 0.66) \times 4.98( \pm 0.14)$ & $4.88 \pm 0.34$ \\
\hline R2LK1 & Pomegranate tree & Wood & Kalykas, Chania & 2014 & $4.78 \pm 0.02$ & white to yellow & $\mathrm{nf}$ & $3.04 \pm 0.20$ \\
\hline $\mathrm{K} 1 \mathrm{KP} 1$ & Kumquat tree & Wood & Agrokipio, Chania & 2014 & $3.71 \pm 0.11$ & white to pale golden & $\mathrm{nf}$ & $2.69 \pm 0.16$ \\
\hline K1LVK1 & Kumquat tree & Wood & Agrokipio, Chania & 2014 & $5.20 \pm 0.02$ & white to light olive-green & $\mathrm{nf}$ & $3.88 \pm 0.32$ \\
\hline K1LK1 & Kumquat tree & Wood & Agrokipio, Chania & 2014 & $2.77 \pm 0.21$ & white to olive-green & $4.78( \pm 0.16) \times 2.06( \pm 0.09)$ & $2.67 \pm 0.07$ \\
\hline K1AL1 & Kumquat tree & Wood & Agrokipio, Chania & 2014 & $10.70 \pm 0.11$ & white & $\mathrm{nf}$ & $4.90 \pm 0.40$ \\
\hline AKKON1LK1 & Silk tree & Wood & Iraklio & 2014 & $4.08 \pm 0.04$ & white to pale golden & $\mathrm{nf}$ & $2.74 \pm 0.13$ \\
\hline MP1 & Apple tree & Wood & Lasithi plateau & 2013 & $3.32 \pm 0.20$ & white to golden & $\mathrm{nf}$ & $2.99 \pm 0.16$ \\
\hline MA2 & Apple tree & Wood & Lasithi plateau & 2013 & $2.06 \pm 0.08$ & white to pale pink & $6.41( \pm 0.25) \times 2.35( \pm 0.11)$ & $2.99 \pm 0.10$ \\
\hline MK1 & Apple tree & Wood & Lasithi plateau & 2013 & $1.95 \pm 0.29$ & white to golden & $\mathrm{nf}$ & $3.29 \pm 0.16$ \\
\hline MSP1 & Apple tree & Wood & Lasithi plateau & 2013 & $2.94 \pm 0.02$ & white to pale pink & $6.53( \pm 0.28) \times 2.68( \pm 0.10)$ & $2.72 \pm 0.08$ \\
\hline MIL2mLK1 & Apple tree & Fruiting body & Lasithi plateau & 2014 & $5.10 \pm 0.11$ & white to pale yellow & nf & $4.55 \pm 0.23$ \\
\hline MIL2LKP1 & Apple tree & Wood & Lasithi plateau & 2014 & $3.04 \pm 0.20$ & white to golden & $\mathrm{nf}$ & $4.48 \pm 0.23$ \\
\hline MOUR1P1 & Mulberry tree & Wood & Lasithi plateau & 2013 & $2.91 \pm 0.21$ & white to pink & $6.78( \pm 0.25) \times 3.63( \pm 0.12)$ & $3.96 \pm 0.20$ \\
\hline MOUR2P3 & Mulberry tree & Wood & Agrokipio, Chania & 2014 & $5.15 \pm 0.05$ & white to pink & $7.32( \pm 0.45) \times 4.30( \pm 0.17)$ & $4.24 \pm 0.17$ \\
\hline MOUR2A1 & Mulberry tree & Wood & Agrokipio, Chania & 2014 & $7.77 \pm 0.06$ & pale pink & $11.78( \pm 1.53) \times 4.73( \pm 0.17)$ & $3.08 \pm 0.14$ \\
\hline MOUR3mKL1 & Mulberry tree & Fruiting body & Iraklio & 2014 & $5.29 \pm 0.04$ & white to pale golden & $\mathrm{nf}$ & $4.11 \pm 0.21$ \\
\hline MOUR4mLK1 & Mulberry tree & Fruiting body & Therissos, Chania & 2014 & $4.57 \pm 0.13$ & white to yellow & $\mathrm{nf}$ & $4.36 \pm 0.26$ \\
\hline AM1KL1 & Almond tree & Wood & Apokoronas, Chania & 2014 & $10.98 \pm 0.02$ & light olive-green & $4.78( \pm 0.17) \times 2.48( \pm 0.10)$ & $3.49 \pm 0.20$ \\
\hline AM1mXL1 & Almond tree & Fruiting body & Apokoronas, Chania & 2014 & $4.48 \pm 0.28$ & dark golden & $\mathrm{nf}$ & $2.61 \pm 0.13$ \\
\hline AM2XL1 & Almond tree & Wood & Apokoronas, Chania & 2014 & $4.51 \pm 0.19$ & dark golden & $\mathrm{nf}$ & $2.67 \pm 0.10$ \\
\hline AL1mLK1 & Tamarisk tree & Fruiting body & Nea hora, Chania & 2014 & $3.08 \pm 0.10$ & pale pink to yellow & $\mathrm{nf}$ & $3.13 \pm 0.12$ \\
\hline MEL1LK1 & Chinaberry tree & Wood & Nea hora, Chania & 2014 & $3.16 \pm 0.02$ & pale yellow & $6.08( \pm 0.23) \times 2.23( \pm 0.05)$ & $2.57 \pm 0.10$ \\
\hline D1LV1 & Loquat tree & Wood & Agios Thomas, Iraklio & 2014 & $16.74 \pm 0.02$ & white & $\mathrm{nf}$ & $3.48 \pm 0.19$ \\
\hline AV1LK3 & Avocado tree & Wood & Nea hora, Chania & 2014 & $2.29 \pm 0.27$ & light to dark brown & $4.28( \pm 0.17) \times 2.83( \pm 0.08)$ & $2.40 \pm 0.09$ \\
\hline AGK2L1 & Jerusalem sage & Wood & Apokoronas, Chania & 2014 & $6.37 \pm 0.21$ & white & nf & $2.70 \pm 0.12$ \\
\hline AGK2mL1 & Jerusalem sage & Fruiting body & Apokoronas, Chania & 2014 & $6.01 \pm 0.42$ & white & $\mathrm{nf}$ & $2.64 \pm 0.12$ \\
\hline AXL1LK1 & Pear tree & Wood & Lasithi plateau & 2014 & $4.05 \pm 0.29$ & white to golden & $\mathrm{nf}$ & $2.46 \pm 0.15$ \\
\hline AXL1SP1 & Pear tree & Wood & Lasithi plateau & 2014 & $2.43 \pm 0.09$ & dark olive-green to gray & $\mathrm{nf}$ & $3.11 \pm 0.16$ \\
\hline KAR1mLP1 & Walnut tree & Fruiting body & Lasithi plateau & 2014 & $3.42 \pm 0.58$ & white to golden & $\mathrm{nf}$ & $3.58 \pm 0.20$ \\
\hline KAR1LEN1 & Walnut tree & Wood & Lasithi plateau & 2014 & $4.11 \pm 0.10$ & white & $\mathrm{nf}$ & $2.63 \pm 0.14$ \\
\hline KAR1LKON1 & Walnut tree & Wood & Lasithi plateau & 2014 & $5.11 \pm 0.15$ & white & $\mathrm{nf}$ & $3.22 \pm 0.20$ \\
\hline PLAT1mL1 & Plane tree & Fruiting body & Chania & 2014 & $8.35 \pm 0.11$ & white & $\mathrm{nf}$ & $3.10 \pm 0.17$ \\
\hline GKFRLV3 & Grapefruit tree & Wood & Agrokipio, Chania & 2014 & $7.48 \pm 0.07$ & white to light pink & $\mathrm{nf}$ & $3.64 \pm 0.26$ \\
\hline ROMP1mLP1 & Black locust tree & Fruiting body & Chania & 2014 & $2.06 \pm 0.05$ & white to golden & $\mathrm{nf}$ & $3.35 \pm 0.21$ \\
\hline K.E. Fomit4 & Olive tree & Wood & Messinia, Kalamata & 2001 & $5.13 \pm 0.02$ & white to golden & $\mathrm{nf}$ & $3.06 \pm 0.28$ \\
\hline
\end{tabular}

${ }^{\mathrm{w}}$ Codes of fungal isolates obtained from various hosts with wood decay symptoms. Fomitiporia mediterranea strain K.E. Fomit4, belonging to Benaki

Phytopathological Institute Collection (BPIC), was provided by Benaki Phytopathological Institute, Kifissia, Athens, Greece, and served as the reference strain.

x Substrate (wood or fruiting body) where fungal strains were isolated.

y Isolates were grown on PDA, at $24^{\circ} \mathrm{C}$ in the dark and the colonies' diameter was measured at day $3,7,10,14,17$, and 21 or stopped earlier, when Petri dish was completely covered.

${ }^{\mathrm{z}} \mathrm{nf}$ indicates that isolates did not form conidia within the 21-day incubation period. 
the inoculation point. Likewise, pruning-wound inoculated stems and branches were cut in November 2015 (7-months post inoculation), the plastic tube was removed, and longitudinal sections through the pruning-wound surface were carried out to measure the extension of symptomatic wood below the inoculation point. To verify the presence of the applied causal agent in diseased wood tissues and confirm pathogenicity in each plant-pathogen pathosystem, reisolations of the pathogens were performed by following the procedure described previously. An isolate was considered pathogenic on a given host when the wood discoloration length above and below the inoculation point in artificially inoculated plants was significantly higher compared with the mock-inoculated ones (control) and the applied fungus was successfully reisolated from artificially inoculated plants, but not from controls.

Statistical analysis. In trunk-drilling inoculated plants, analysis of variance (ANOVA) was employed to evaluate the pathogenic effects of the fungal strains on wood discoloration length above and below the inoculation point. When a significant $F$ test was obtained for treatments $(P<0.05)$, the data were subjected to means separation by Tukey's test and standard errors of means were also calculated. Morphological and physiological characteristics of $F$. mediterranea isolates were also analyzed by Tukey's test $(P<0.05)$ and by determining the Pearson's $r$ correlation coefficients $(P<0.05$ or 0.01$)$. Moreover, in pruning-wood inoculated plants, data on wood discoloration length below the inoculation point were analyzed by carrying out a two-sample t test $(P<0.05)$.

\section{Results}

Morphological, physiological and molecular identification of fungal isolates. Morphological and physiological characteristics of 58 fungal strains isolated from 18 plant species showing typical wood decay symptoms are presented in Table 1. Among Ascomycota isolates, colony color was indicative of their holotypes. All isolated ascomycetes formed conidia of various sizes within the 21-day incubation period examined, apart from Disymoshpaeria, Eutypa, and Phialocephala strains. Among Ascomycota members, Phialophora

Table 2. Phylogenetic relatives of the 58 fungal isolates obtained from 18 plant species and exhibited typical wood decay symptoms

\begin{tabular}{|c|c|c|c|c|c|c|}
\hline OTU & Isolate & Host (substrate) & Phylogenetic relative & Similarity $(\%)$ & Isolation source & Reference \\
\hline \multicolumn{7}{|c|}{ Ascomycota; Pezizomycotina; Sordariomycetes; Hypocreomycetidae; Hypocreales; Nectriaceae } \\
\hline 1 & R1K1, MEL1LK1 & $\begin{array}{l}\text { Pomegranate tree } \\
\text { (Wood), Chinaberry } \\
\text { tree (Wood) }\end{array}$ & $\begin{array}{l}\text { strain } 212 \mathrm{~d} / \text { Xenoacremonium } \\
\text { falcatum CBS } 400.85 / X \text {. } \\
\text { recifei } \text { CBS } 541.89\end{array}$ & $\begin{array}{l}98.8-100 / 99.0-99.4 / \\
99.0-99.4\end{array}$ & $\begin{array}{l}\text { endophyte from roots of } \\
\text { eastern cottonwood/ } \\
\text { Pinus radiata/soil, } \\
\text { Brazil }\end{array}$ & $\begin{array}{l}\text { JX244031/Lombard et al. } \\
\text { (2015)/Lombard et al. } \\
\text { (2015) }\end{array}$ \\
\hline 2 & MA2, MSP1 & Apple tree (Wood) & $\begin{array}{l}\text { Cosmospora sp. OT1A-312b/ } \\
\text { Cosmospora sp. IMI } \\
\text { 362240/Pseudocosmospora } \\
\text { rogersonii G.J.S. 09-1384 }\end{array}$ & $99.9 / 95.2 / 95.2$ & $\begin{array}{l}\text { leaves of Verdeal- } \\
\text { Transmontana olive } \\
\text { cultivar/stem tissue of } \\
\text { Vitis/Eutypella } \mathrm{sp} .\end{array}$ & $\begin{array}{l}\text { KT804138/KJ676169/ } \\
\text { Herrera et al. (2013) }\end{array}$ \\
\hline 3 & $\begin{array}{l}\text { MOUR1P1, MOUR2P3, } \\
\text { MOUR2A1 }\end{array}$ & Mulberry tree (Wood) & $\begin{array}{l}\text { Fusarium solani UOC/PTS/ } \\
\text { RDWW-22 }\end{array}$ & $99.6-100$ & $\begin{array}{l}\text { endophyte-Calamus } \\
\text { thwaitesii }\end{array}$ & KT150278 \\
\hline \multicolumn{7}{|c|}{ Ascomycota; Pezizomycotina; Sordariomycetes; Sordariomycetidae; Diaporthales; Togniniaceae } \\
\hline 4 & A5LKK1 & Grapevine (Wood) & $\begin{array}{l}\text { Phaeoacremonium minimum } \\
\text { CBS } 100548\end{array}$ & 100 & Olea europaea, Italy & Groenewald et al. (2001) \\
\hline \multicolumn{7}{|c|}{ Ascomycota; Pezizomycotina; Sordariomycetes; Sordariomycetidae; Calosphaeriales; Pleurostomataceae } \\
\hline 5 & E1PK1, AV1LK3 & $\begin{array}{l}\text { Olive tree (Wood), } \\
\text { Avocado tree (Wood) }\end{array}$ & $\begin{array}{l}\text { Pleurostomophora } \\
\quad \text { richardsiae IFM41579/ } \\
\text { Pleurostomophora } \\
\text { richardsiae CBS } 117177\end{array}$ & $99.6 / 99.4$ & clinical/grapevine & AB364695/EF042106 \\
\hline \multicolumn{7}{|c|}{ Ascomycota; Pezizomycotina; Sordariomycetes; Sordariomycetidae; Cephalothecaceae } \\
\hline 6 & K1LK1 & Kumquat tree (Wood) & $\begin{array}{l}\text { strain 695_ICIPE/ } \\
\text { Phialemonium } \\
\text { dimorphosporum } \mathrm{E} 9430 \mathrm{~h}\end{array}$ & $99.8 / 99.6$ & $\begin{array}{l}\text { seed-born fungal } \\
\text { endophyte/endophytes- } \\
\text { rainforest plant }\end{array}$ & KM463111/JN559402 \\
\hline \multicolumn{7}{|c|}{ Ascomycota; Pezizomycotina; Sordariomycetes; Xylariomycetidae; Xylariales; Diatrypaceae } \\
\hline 7 & GKFRLV3 & Grapefruit tree (Wood) & $\begin{array}{l}\text { Eutypa leptoplaca CBS } \\
\quad 286.87\end{array}$ & 99.8 & grapevine pathogen & $\begin{array}{l}\text { Trouillas and Gubler } \\
\text { (2004) }\end{array}$ \\
\hline \multicolumn{7}{|c|}{ Ascomycota; Pezizomycotina; Dothideomycetes; Dothideomycetidae; Dothideales; Saccotheciaceae } \\
\hline 8 & R1YKL1 & Pomegranate tree (Wood) & $\begin{array}{l}\text { Aureobasidium pullulans } \\
\text { CBS } 584.75\end{array}$ & 100 & Vitis vinifera & KT693733 \\
\hline \multicolumn{7}{|c|}{ Ascomycota; Pezizomycotina; Dothideomycetes; Pleosporomycetidae; Pleosporales; Didymosphaeriaceae } \\
\hline 9 & K1LVK1, A5LV1 & $\begin{array}{l}\text { Kumquat tree (Wood), } \\
\text { Grapevine (Wood) }\end{array}$ & $\begin{array}{l}\text { Didymosphaeria variabile } \\
\text { STE-U } 6311\end{array}$ & & $\begin{array}{l}\text { stone fruit trees and } \\
\text { woody hosts }\end{array}$ & Damm et al. (2008) \\
\hline \multicolumn{7}{|c|}{ Ascomycota; Pezizomycotina; Eurotiomycetes; Chaetothyriomycetidae; Chaetothyriales; Herpotrichiellaceae } \\
\hline 10 & AM1KL1 & Almond tree (Wood) & $\begin{array}{l}\text { Phialophora alba ICMP: } \\
17034\end{array}$ & 100 & $\begin{array}{l}\text { forests and kiwifruit } \\
\text { orchards }\end{array}$ & Johnston et al. (2010) \\
\hline \multicolumn{7}{|c|}{ Ascomycota; Pezizomycotina; Eurotiomycetes; Chaetothyriomycetidae; Phaeomoniellales; Phaeomoniellaceae } \\
\hline 11 & A2LP1, A5LP1 & Grapevine (Wood) & $\begin{array}{l}\text { Phaeomoniella } \\
\text { chlamydospora ICMP: } \\
17117\end{array}$ & 99.6-100 & Vitis vinifera & HM116754 \\
\hline \multicolumn{7}{|c|}{ Ascomycota; Pezizomycotina; Leotiomycetes; Leotiomycetidae; Helotiales; Vibrisseaceae } \\
\hline 12 & AXL1SP1 & Pear tree (Wood) & $\begin{array}{l}\text { Phialocephala } \\
\text { dimorphospora } \mathrm{CBS} \\
300.62\end{array}$ & 99.0 & slime in pulp mill & Grünig et al. (2002) \\
\hline \multicolumn{7}{|c|}{ Basidiomycota; Agaricomycotina; Agaricomycetes; Polyporales; Polyporaceae } \\
\hline 13 & D1LV1 & Loquat tree (Wood) & Trametes versicolor $\mathrm{X}-29$ & 99.8 & saprophyte from soil & Thorn et al. (1996) \\
\hline 14 & PLAT1mL1 & Plane tree (Fruiting body) & $\begin{array}{l}\text { Trametes ljubarskyi PRM } \\
\quad 622107\end{array}$ & 99.5 & Prunus cerasus & Tomšovský et al. (2006) \\
\hline \multicolumn{7}{|c|}{ Basidiomycota; Agaricomycotina; Agaricomycetes; Polyporales; Polyporaceae } \\
\hline 15 & AGK2L1, AGK2mL1 & $\begin{array}{l}\text { Jerusalem sage (Wood), } \\
\text { Jerusalem sage } \\
\text { (Fruiting body) }\end{array}$ & $\begin{array}{l}\text { Perenniporia truncatospora } \\
\text { Cui 6987/P. tenuis } \text { Cui } \\
5523\end{array}$ & $98.0 / 98.0$ & $\begin{array}{l}\text { fruiting body/fruiting } \\
\text { body }\end{array}$ & $\begin{array}{l}\text { Zhao and Cui (2013)/ } \\
\text { Zhao and Cui (2012) }\end{array}$ \\
\hline \multicolumn{7}{|c|}{ Basidiomycota; Agaricomycotina; Agaricomycetes; Auriculariales; Auriculariaceae } \\
\hline 16 & KAR1LEN1 & Walnut tree (Wood) & $\begin{array}{l}\text { Auricularia auricula-judae } \\
\text { LYBR } 5404\end{array}$ & 99.3 & leaves (Feuillu) & KP776003 \\
\hline
\end{tabular}

\footnotetext{
$\mathrm{z}$ The reference strain K.E. Fomit4 was also included.
} 
and Phaeoacremonium exhibited the highest and lowest growth rate, respectively. The hyphal width of the isolated ascomycetes varied between 1.98 and $4.88 \mu \mathrm{m}$. All Basidiomycota isolates also had the typical colony color of their holotypes. Their growth rate varied widely from 1.89 to $16.74 \mathrm{~mm} /$ day, while their hyphal width ranged between 2.28 and $4.90 \mu \mathrm{m}$ (Table 1 ).

By 5.8S rRNA gene-ITS sequencing analysis, the fungal isolates were classified into 25 operational taxonomic units (OTUs), which were equally split into the phyla Ascomycota and Basidiomycota (Table 2). In particular, OTUs nos. 1 to 12 were ascomycetes belonging to the genera Xenoacremonium, Cosmospora, Fusarium, Phaeoacremonium, Pleurostomophora, Phialemonium, Eutypa, Aureobasidium, Didymosphaeria, Phialophora, Phaeomoniella, and Phialocephala, whereas OTUs nos. 13 to 25 were basidiomycetes affiliated with Trametes, Perenniporia, Auricularia, Aphanobasidium, Coniophora, Fuscoporia, Inonotus, Phellinus, Inocutis, and Fomitiporia spp. (Table 2; Figs. 1 and 2). Apart from isolate E1PK1, which was related to Pleurostomophora richardsiae, no other ascomycetes were found to cause wood tissue discoloration of olive tree in the present study. Fomitiporia mediterranea was the major causal agent of grapevine wood decay, with the exception of the Kolimpari specimen where the infection was attributed only to a mixture of ascomycetes strains. Considering further host specificity, chinaberry (Melia azedarach L.), avocado, and grapefruit (Citrus paradisi L.) trees were infected by ascomycetes, whereas loquat (Eriobotrya japonica L.), plane, walnut (Juglans regia L.), black locust, tamarisk (Tamarix parviflora DC.), and orange trees as well as Jerusalem sage (Phlomis fruticosa L.) were susceptible to Agaricomycetes rot pathogens; however, the number of these plant samples infected was marginal to permit reliable conclusions. Among these isolates, important phytopathogenic strains of the genera Fomitiporia, Inonotus, Phellinus, Inocutis, Fuscoporia, Trametes, Fusarium, Eutypa, Phaeomoniella, Phaeoacremonium, and Pleurostomophora were included. The white rot basidiomycetes $F$. mediterranea (20 isolates out of 58, 34.5\%) and I. hispidus (6 isolates out of 58, 10.3\%) were the most predominant (Table 2). In particular, F. mediterranea and I. hispidus strains affected 19 and 5 of the samples examined, respectively (Tables 1 and 2). In most cases, only a single fungal taxon was found in each sample; however, two or three fungal species were recovered from 14 out of 38 samples collected. More rarely, two fungal taxa were observed to emerge simultaneously from the same wood chip, i.e., F. mediterranea/P. chlamydospora and $P$. chlamydospora/Phaeoacremonium minimum W. Gams, Crous, M.J. Wingf. \& Mugnai in grapevine tissues, and $F$. mediterranea/P. richardsiae in olive tree tissues. Among the 21 $F$. mediterranea isolates sequenced (including the reference strain K.E.

Table 2. (continued from preceding page)

\begin{tabular}{|c|c|c|c|c|c|c|}
\hline OTU & Isolate & Host (substrate) & Phylogenetic relative & Similarity $(\%)$ & Isolation source & Reference \\
\hline \multicolumn{7}{|c|}{ Basidiomycota; Agaricomycotina; Agaricomycetes; Agaricomycetidae; Agaricales; Pterulaceae } \\
\hline 17 & KAR1LKON1 & Walnut tree (Wood) & $\begin{array}{l}\text { Aphanobasidium } \\
\text { pseudotsugae CFMR: } \\
\text { HHB- } 822\end{array}$ & 82.9 & Quercus log & Binder et al. (2010) \\
\hline \multicolumn{7}{|c|}{ Basidiomycota; Agaricomycotina; Agaricomycetes; Agaricomycetidae; Boletales; Coniophoraceae } \\
\hline 18 & K1AL1 & Kumquat tree (Wood) & $\begin{array}{l}\text { strain } \mathrm{C} 1 / \text { Coniophora } \\
\text { marmorata } \mathrm{P} 158 / C \\
\text { olivacea } \mathrm{P} 151\end{array}$ & 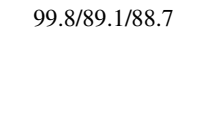 & $\begin{array}{l}\text { wood rot of citrus/mining } \\
\text { timber } 1952 / \text { brown-rot } \\
\text { fungi-decay in } \\
\text { buildings woodwork }\end{array}$ & $\begin{array}{l}\text { Roccotelli et al. (2014)/ } \\
\text { Schmidt et al. (2002)/ } \\
\text { Schmidt et al. (2002) }\end{array}$ \\
\hline \multicolumn{7}{|c|}{ Basidiomycota; Agaricomycotina; Agaricomycetes; Hymenochaetales; Hymenochaetaceae } \\
\hline 19 & E11mLK1 & Olive tree (Fruiting body) & Fuscoporia torulosa 759 & 100 & $\begin{array}{l}\text { wood rot fungus of } \\
\text { standing trees }\end{array}$ & Guglielmo et al. (2007) \\
\hline 20 & $\begin{array}{l}\text { MP1, MOUR4mLK1, } \\
\text { MK1, MIL2mLK1, } \\
\text { MIL2LKP1, } \\
\text { KAR1mLP1 }\end{array}$ & $\begin{array}{l}\text { Apple tree (Wood), } \\
\text { Mulberry tree (Fruiting } \\
\text { body), Apple tree } \\
\text { (Wood), Apple tree } \\
\text { (Fruiting body), Apple } \\
\text { tree (Wood), Walnut } \\
\text { tree (Fruiting body) }\end{array}$ & Inonotus hispidus 15 & $98.3-99.1$ & $\begin{array}{l}\text { wood decay fungus of } \\
\text { Malus sp. }\end{array}$ & Schmidt et al. (2012) \\
\hline 21 & MOUR3mKL1 & $\begin{array}{l}\text { Mulberry tree (Fruiting } \\
\text { body) }\end{array}$ & Inonotus rickii IFP 10359 & 99.0 & $\begin{array}{l}\text { canker and decay of } \\
\text { Hevea brasiliensis }\end{array}$ & Dai et al. (2010) \\
\hline 22 & ROMP1mLP1 & $\begin{array}{l}\text { Black locust tree (Fruiting } \\
\text { body) }\end{array}$ & $\begin{array}{l}\text { Phellinus rimosus/Fulvifomes } \\
\quad \text { (ex Phellinus) merrillii } \\
\text { PM950703-1 }\end{array}$ & $95.3 / 88.3$ & NR/NR & JX484016/EU035311 \\
\hline 23 & AM1mXL1, AM2XL1 & $\begin{array}{l}\text { Almond tree (Fruiting } \\
\text { body), Almond tree } \\
\text { (Wood) }\end{array}$ & $\begin{array}{l}\text { Phellinus sp. } 1 \text { DE-2016/ } \\
\text { Phellinus pomaceus } \text { TN- } \\
449\end{array}$ & 98.6-98.8/98.3-98.5 & $\begin{array}{l}\text { fungus from deciduous- } \\
\text { peach/white trunk rot of } \\
\text { hardwoods-Prunus } \\
\text { domestica }\end{array}$ & $\begin{array}{l}\text { KU863540/Brazee } \\
\quad(2015)\end{array}$ \\
\hline 24 & AL1mLK1 & $\begin{array}{l}\text { Tamarisk tree (Fruiting } \\
\text { body) }\end{array}$ & $\begin{array}{l}\text { Inocutis tamaricis HAI 1291/ } \\
\text { Inocutis tamaricis 6-27 }\end{array}$ & $99.0 / 95.8$ & $\begin{array}{l}\text { NR/pathogen of Tamarix } \\
\text { chinensis }\end{array}$ & GQ253453/HM050416 \\
\hline 25 & $\begin{array}{l}\text { K1KP1, R1LK1, } \\
\text { R1LKP1, E2mXL1, } \\
\text { E2LK3, A3LKP2, } \\
\text { A4LKP1, E7LK1, } \\
\text { P1KP1, P2KP1, } \\
\text { AKKON1LK1, } \\
\text { E9mLK1, E10mLK1, } \\
\text { AXL1LK1, A2LKP1, } \\
\text { E6LK1, E5LK1, } \\
\text { R2LK1, E1KP1, K.E. } \\
\text { Fomit4 }{ }^{\mathrm{z}}, \text { E3LK1 }\end{array}$ & $\begin{array}{l}\text { Kumquat tree (Wood), } \\
\text { Pomegranate tree } \\
\text { (Wood), Pomegranate } \\
\text { tree (Wood), Olive tree } \\
\text { (Fruiting body), Olive } \\
\text { tree (Wood), Grapevine } \\
\text { (Wood), Grapevine } \\
\text { (Wood), Olive tree } \\
\text { (Wood), Orange tree } \\
\text { (Wood), Orange tree } \\
\text { (Wood), Silk tree } \\
\text { (Wood), Olive tree } \\
\text { (Fruiting body), Olive } \\
\text { tree (Fruiting body), } \\
\text { Pear tree (Wood), } \\
\text { Grapevine (Wood), } \\
\text { Olive tree (Wood), } \\
\text { Olive tree (Fruiting } \\
\text { body), Pomegranate } \\
\text { tree (Wood), Olive tree } \\
\text { (Wood), Olive tree } \\
\text { (Wood), Olive tree } \\
\text { (Wood) }\end{array}$ & $\begin{array}{l}\text { Fomitiporia mediterranea } 45 \text { / } \\
\quad 23.3\end{array}$ & $98.7-99.6$ & $\begin{array}{l}\text { wood decay fungus- } \\
\text { grapevine }\end{array}$ & Fischer (2002) \\
\hline
\end{tabular}


Fomit4), eight distinct genotypes were identified, with half of them being identical in ITS-5.8S rRNA gene with previously deposited sequences (Fig. 2). The other four genotypes were distinct and reported for the first time, showing phylogenetic relatedness to known phytopathogenic strains of the species $F$. mediterranea (Fischer 2002; Roccotelli et al. 2014). All $F$. mediterranea strains isolated in the present study showed 98.7 to $99.6 \%$ similarity with strain $45 / 23$, which is the holotype of F. mediterranea (Fischer 2002).

Physiological and morphological characterization of Fomitiporia mediterranea isolates. Analysis of variance revealed that $F$. mediterranea isolates differed significantly in terms of growth rate ( $\mathrm{df}=20, F=$ 51.99, $P<00.001$ ), pigmentation on the upper side $(\mathrm{df}=20, F=15.56$, $P<0.001)$ and underside $(\mathrm{df}=20, F=37.84, P<0.001)$ of PDA plates, and mycelium abundance ( $\mathrm{df}=20, F=30.14, P<0.001)$ (Fig. 3).
However, no significant differences among isolates were observed in hyphae width $(\mathrm{df}=20, F=1.48, P=0.083)$. The average growth rate values of $F$. mediterranea isolates ranged from 1.89 to $6.23 \mathrm{~mm} /$ day, while pigmentation values on the upper side and the underside of PDA plates ranged from 0.5 to 4.0 and 0.0 to 3.5, respectively. Among the $21 \mathrm{~F}$. mediterranea isolates assessed (the reference strain K.E. Fomit4 was also included), eight were designated as S- and 13 as Btype, according to Fischer (2006). In addition, mycelium abundance of the isolates varied within 1.0 (sparse development of aerial hyphae) and 4.0 (intensive woolly mycelium), whereas isolates' hyphae width ranged between 2.28 and $3.06 \mu \mathrm{m}$. Moreover, the growth rate of isolate E9mLK1 was the greatest, followed by strain K.E. Fomit4, while the respective rates of isolates E3LK1, E5LK2, and A2LKP1 were the lowest $(P<0.05)$. Interestingly, these three isolates exhibiting the slowest

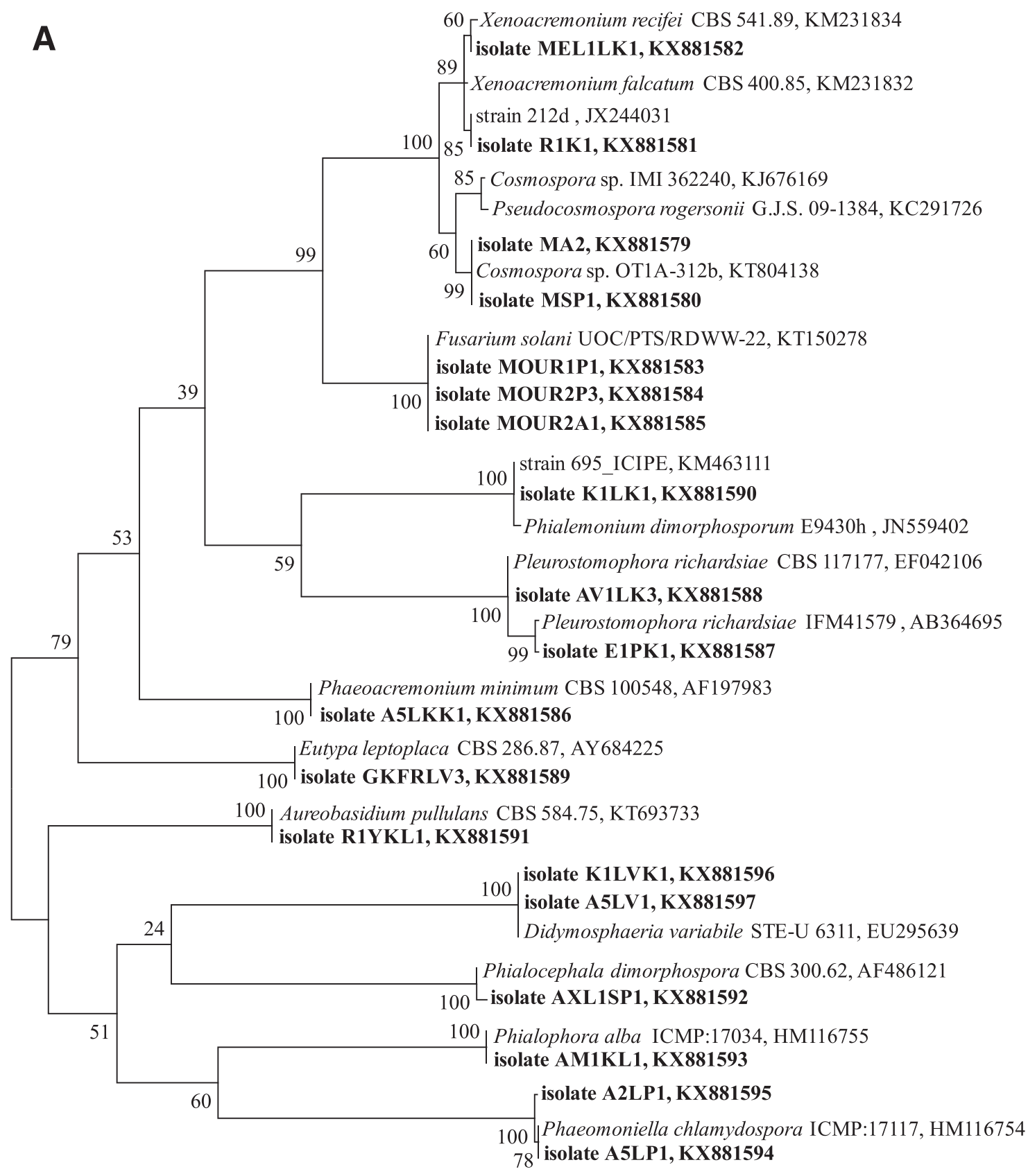

$\mathbf{0 . 0 5 0}$

Continued on following page

Fig. 1. Phylogenetic allocation of ascomycetes A, and basidiomycetes (other than Fomitiporia) B, isolated from plant species, showing typical wood decay symptoms. 
growth rates were placed in distinct genotypes. Apart from the growth rate, no other relationships among morphological and physiological traits, host specificity, and phylogeny were found. Moreover, hyphae width and growth rate were negatively correlated with pigmentation on the upper side $(\mathrm{r}=-0.459, P<0.05)$ and underside $(\mathrm{r}=-0.473$, $P<0.05)$, respectively. A positive correlation between pigmentation on the upper side and underside was also identified $(\mathrm{r}=0.467, P<0.05)$. This indicates that pigment formation was less in the fast-growing fungal strains isolated. In addition, mycelium abundance was highly correlated with the pigmentation on the upper side and underside $(r>0.600$, $P<0.01)$.

Pathogenicity of fungal isolates. After a 7-month incubation period, longitudinal and transverse sections of sampled trunks revealed statistically significant wood tissue discoloration in all trunk-drilling inoculated trees treated with isolates originated from the same plant species compared with the controls (Table 3). Typical internal symptoms were developed as dark brown lesions around the inoculation point (Fig. 4A, E, F, and G). In the cases of the olive-F. mediterra$n e a$ and the grapevine- $F$. mediterranea pathosystems, a light-colored rotten area around the infection hole, similar to those observed under natural infection conditions, was occasionally noticed. On the other hand, control plants exhibited a brown wood discoloration limited mostly around the inoculation site. All fungal isolates tested were pathogenic on the plant species that had been obtained as the extension of wood tissue discoloration above and below the inoculation point in grapevine, olive, pomegranate, kumquat, pear, apple, almond, avocado, and mulberry tree was significantly higher compared with their mock-inoculated applications (Table 4). Moreover, the applied fungus in each plant-pathogen pathosystem was successfully reisolated from the artificially inoculated trees, but not from controls, thus confirming pathogenicity. In addition, $F$. mediterranea isolate A2LKP1 from grapevine was also pathogenic on olive, but

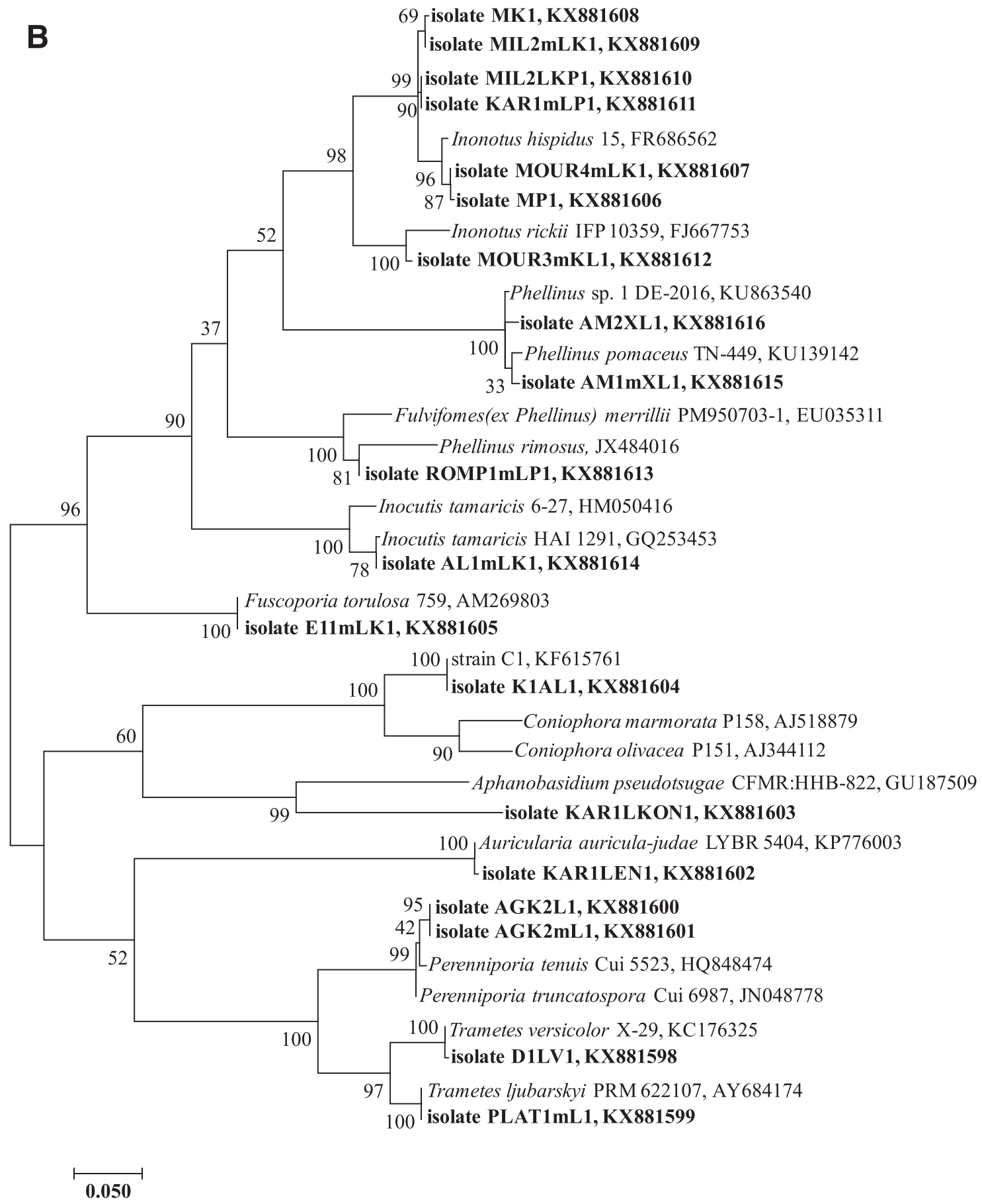

Fig. 1. Continued from previous page. 
nonpathogenic on pomegranate, kumquat, apple, and almond tree. No significant differences in wood discoloration length above and below the inoculation point were observed within each plant species (Table 4).

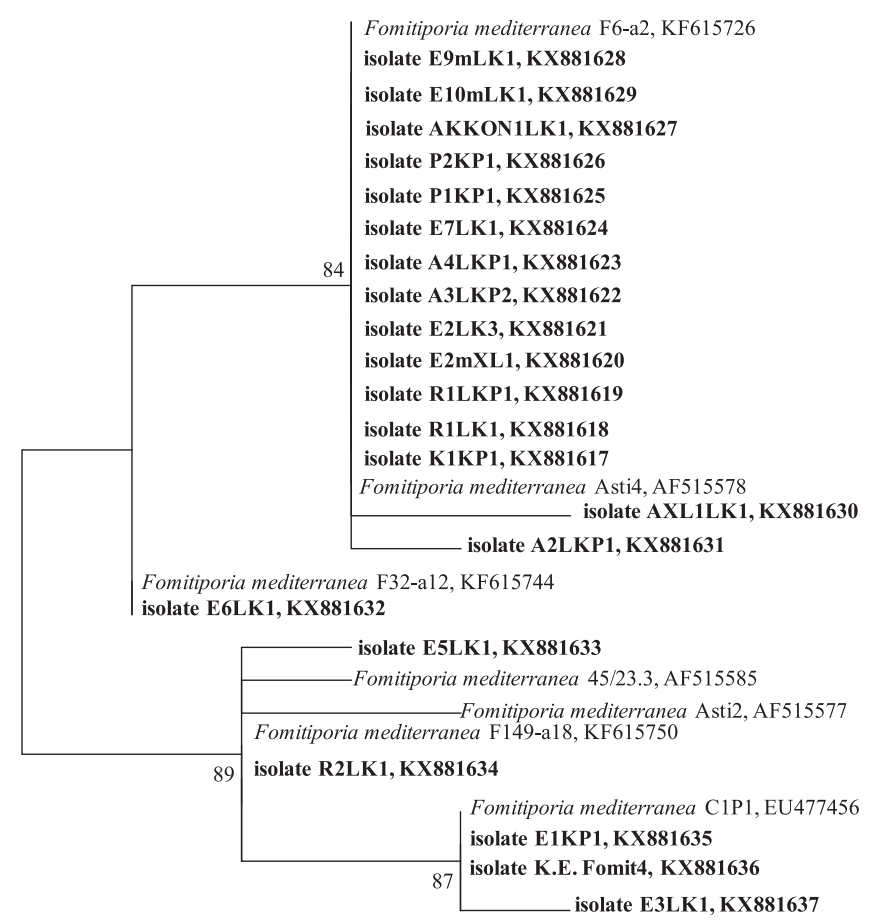

0.0010

Fig. 2. Phylogenetic position of 21 Fomitiporia mediterranea strains, including the reference strain K.E. Fomit4, isolated from 18 plant species, which exhibited extended pathogenicity to their hosts.
In the case of the pruning wound-inoculated trees, a typical lightcolored rotten wood zone adjacent to the inoculation point in stems and branches was commonly observed 7 months after inoculation, followed by a brown-hard-necrotic wood area extending downward (Fig. 4B-D). At this period, a light-yellow mycelium was visible onto the wound surface and inside the wood due to fungal colonization. In control plants, a less-extended necrotic area without either wood rot or colonizing mycelium was observed. The length of wood discoloration below the inoculation site in stems and branches of silk tree, olive, pomegranate, kumquat, and grapevine inoculated with $F$. mediterranea isolates AKKON1LK1, E3LK1, R1LK1, K1KP1, and E3LK1, respectively, was significantly higher than in control plants (Fig. 5). Furthermore, $F$. mediterranea was steadily reisolated from all inoculated plants, but not from controls; therefore, pathogenicity of isolates was verified.

\section{Discussion}

In recent years, increased incidence of wood decay diseases in cultivated and ornamental tree species has been observed worldwide. This increase could be attributed to the more thorough study of the wood-decay pathogens in the recent years than earlier. Among plant species affected frequently are grapevine, olive, citrus, apple, and pomegranate trees. To a lesser extent, other plant species are also noticed to exhibit typical wood rot and decline symptoms, leading occasionally to tree death. Although the causal agents of these diseases have been sufficiently determined in several hosts (Di Marco et al. 2004; Elena et al. 2006; Elena and Paplomatas 2002; Fischer 2006; Paplomatas et al. 2006a, b; Pilotti et al. 2005; 2010; Roccotelli et al. 2014), the etiology of such decay on others still remains unknown. In the present study, a two-year survey was conducted to identify fungi associated with wood decay of tree species.

The isolation method followed here was efficient enough to obtain the three most prominent fungal species associated with esca disease in grapevine, i.e., F. mediterranea, $P$. chlamydospora, and $P$. minimum (Sparapano et al. 2000), and the main causal agent ( $F$. mediterranea) of heart wood rot in olive (Paplomatas et al. 2006a, b) and orange trees (Elena et al. 2006) in Greece. Fomitiporia mediterranea was also isolated from diseased pear, kumquat, pomegranate, and silk
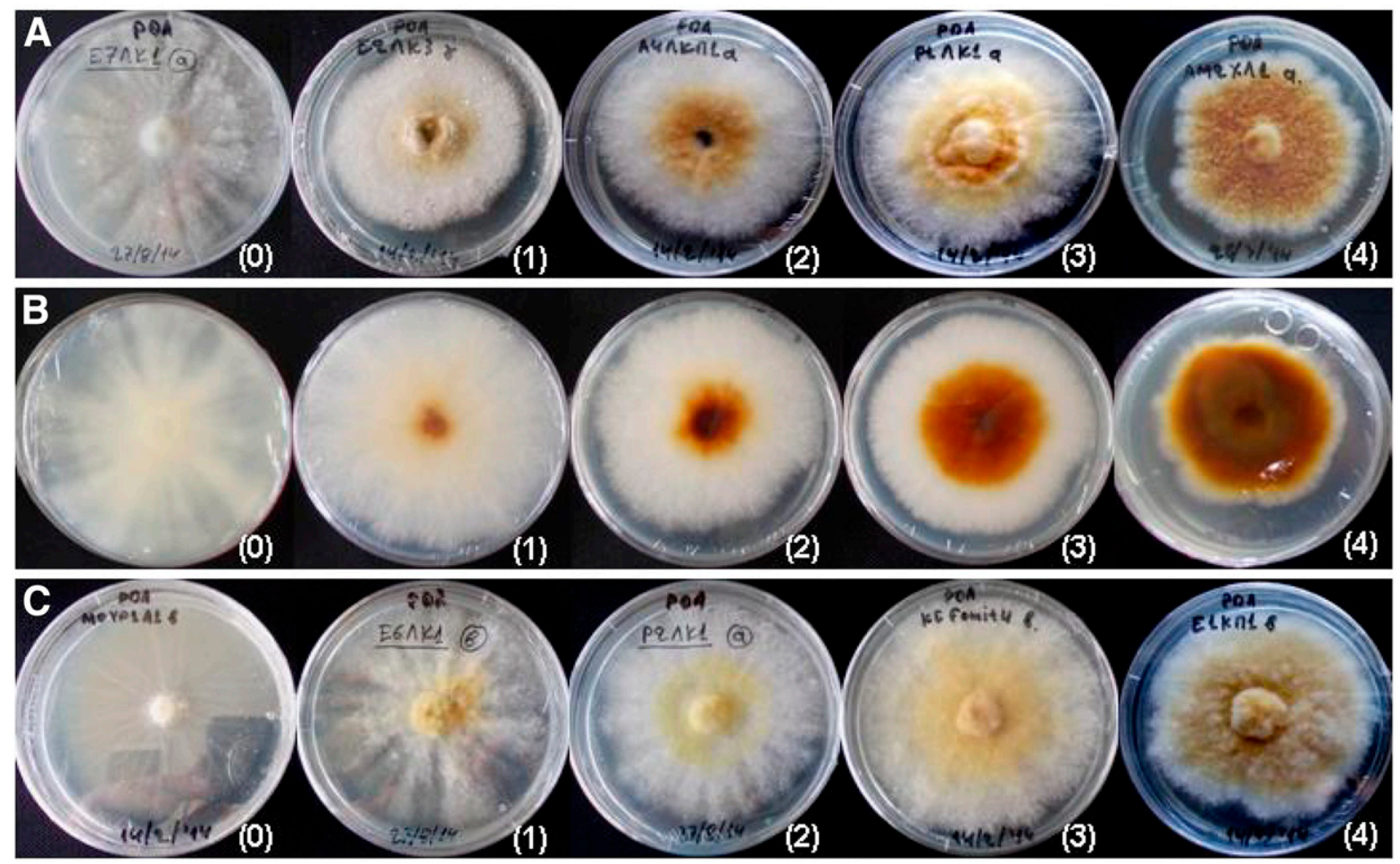

Fig. 3. Differential morphological characteristics of Fomitiporia mediterranea isolates grown on PDA cultures. Pigmentation was visually assessed in a five-point evaluation system regarding the percentage of pigmented area with reference to the entire culture surface on the underside $\mathbf{A}$, and upper side $\mathbf{B}$, of PDA cultures $(0=$ white colony, $1=1$ to $25 \%, 2=26$ to $50 \%, 3=51$ to $75 \%$, and $4=76$ to $100 \%$ of culture surface pigmented). C, Mycelium abundance of fungal cultures was based on a 0 to 4 visual scale $(0=$ yeast-like appearance, $1=$ sparse development of aerial hyphae, 2 = cottony mycelial culture, 3 = modest woolly appearance, and 4 = intensive woolly mycelium). 
trees for the first time worldwide. Artificial inoculation experiments with $F$. mediterranea derived from these hosts verified the pathogenicity of isolates on the plant species that were originated, indicating a further extension of $F$. mediterranea host range list, including hereafter pear, pomegranate, kumquat, and silk tree.

It has been previously hypothesized that $F$. mediterranea inocula reach vineyards from external sources such as old vineyards nearby or carpophores that have been developed on non-Vitis hosts (Surico et al. 2006; 2008). However, no experimental work has been conducted to support such hypothesis (Surico et al. 2006). Cross-pathogenicity tests with $F$. mediterranea conducted here revealed that isolate E3LK1 originated from olive was pathogenic on olive and grapevine. Moreover, $F$. mediterranea isolate A2LKP1 from grapevine was pathogenic on grapevine and olive, but not on pomegranate, kumquat, apple and almond trees, suggesting a partial host range specificity of the fungus. Therefore, the potential efficiency of $F$. mediterranea to be dispersed and transinfect hosts other than that originated in nature should not be excluded. This hypothesis is strengthened by farmers' reports that olive and citrus orchards with high disease incidence are either neighbored with esca-infected vineyards or they have been established in discontinued vineyards shown severe esca symptoms (Elena et al. 2006; present study). Other researchers have likewise proposed host specificity or host-specific cryptic species within $F$. mediterranea, on the basis of pathogenicity tests on citrus trees with $F$. mediterranea isolates from citrus and noncitrus hosts (Elena et al. 2006; Pilotti et al. 2010; Roccotelli et al. 2014).

In contrast to other plant-pathogen pathosystems, artificial inoculation experiments with $F$. mediterranea on tree species and grapevine

Table 3. Analysis of variance for wood discoloration length above and below the inoculation point for grapevine, olive, pomegranate, kumquat, pear, apple, almond, avocado, mulberry and silk tree, artificially inoculated with the isolates A2LKP1-A2LP1, E3LK1-E1PK1-A2LKP1, R1LK1-A2LKP1, K1KP1A2LKP1, AXL1LK1, MP1-A2LKP1, AM2XL1-A2LKP1, AV1LK3, MOYR2P3, and AKKON1LK1 or mock inoculated controls, respectively

\begin{tabular}{|c|c|c|c|c|c|c|c|c|c|c|}
\hline \multirow[b]{2}{*}{ Source } & \multicolumn{10}{|c|}{ df $^{\mathrm{y}}, \boldsymbol{F}$ values $^{\mathrm{z}}$} \\
\hline & Grapevine & $\begin{array}{c}\text { Olive } \\
\text { tree }\end{array}$ & $\begin{array}{c}\text { Pomegranate } \\
\text { tree }\end{array}$ & $\begin{array}{c}\text { Kumquat } \\
\text { tree }\end{array}$ & $\begin{array}{c}\text { Pear } \\
\text { tree }\end{array}$ & $\begin{array}{c}\text { Apple } \\
\text { tree }\end{array}$ & $\begin{array}{c}\text { Almond } \\
\text { tree }\end{array}$ & $\begin{array}{c}\text { Avocado } \\
\text { tree }\end{array}$ & $\begin{array}{c}\text { Mulberry } \\
\text { tree }\end{array}$ & $\begin{array}{l}\text { Silk } \\
\text { tree }\end{array}$ \\
\hline & \multicolumn{10}{|c|}{ Wood discoloration length above the inoculation point } \\
\hline Replication & $2,2.05$ & $2,2.53$ & $2,1.17$ & $2,0.05$ & $2,0.30$ & $2,0.04$ & $2,0.13$ & $2,0.11$ & $2,1.54$ & - \\
\hline Treatment & $2,58.16^{* * *}$ & $3,19.58 * * *$ & $2,16.84 * * *$ & $2,9.46 * *$ & $1,24.74 * * *$ & $2,11.54 * *$ & $2,21.54 * * *$ & $1,27.83 * * *$ & $1,647.78^{* * *}$ & - \\
\hline \multirow{2}{*}{$\begin{array}{c}\text { Replication } \times \\
\text { treatment }\end{array}$} & $4,0.89$ & $6,0.88$ & $4,0.64$ & $4,0.39$ & $2,0.30$ & $4,0.24$ & $4,0.07$ & $2,0.11$ & $2,1.54$ & - \\
\hline & \multicolumn{10}{|c|}{ Wood discoloration length below the inoculation point } \\
\hline Replication & $2,0.02$ & $2,0.33$ & $2,1.33$ & $2,0.29$ & $2,0.22$ & $2,0.83$ & $2,0.01$ & $2,0.07$ & $2,1.03$ & $2,0.93$ \\
\hline Treatment & $2,26.04 * * *$ & $3,7.57 * * *$ & $2,10.80 * *$ & $2,11.22 * *$ & $1,26.62 * * *$ & $2,18.35^{* * *}$ & $2,45.37 * * *$ & $1,29.78 * * *$ & $1,34.83 * * *$ & $1,27.23 * * *$ \\
\hline $\begin{array}{c}\text { Replication } \times \\
\text { treatment }\end{array}$ & $4,0.08$ & $6,0.53$ & $4,0.52$ & $4,0.77$ & $2,0.22$ & $4,1.32$ & $4,0.07$ & $2,0.07$ & $2,1.03$ & $2,0.93$ \\
\hline
\end{tabular}

${ }^{y}$ Degrees of freedom among groups.

${ }^{\mathrm{z}}$ Symbols $* *$ and $* * *$ indicate significance at $P<0.01$ and 0.001 , respectively, according to the $F$ test.
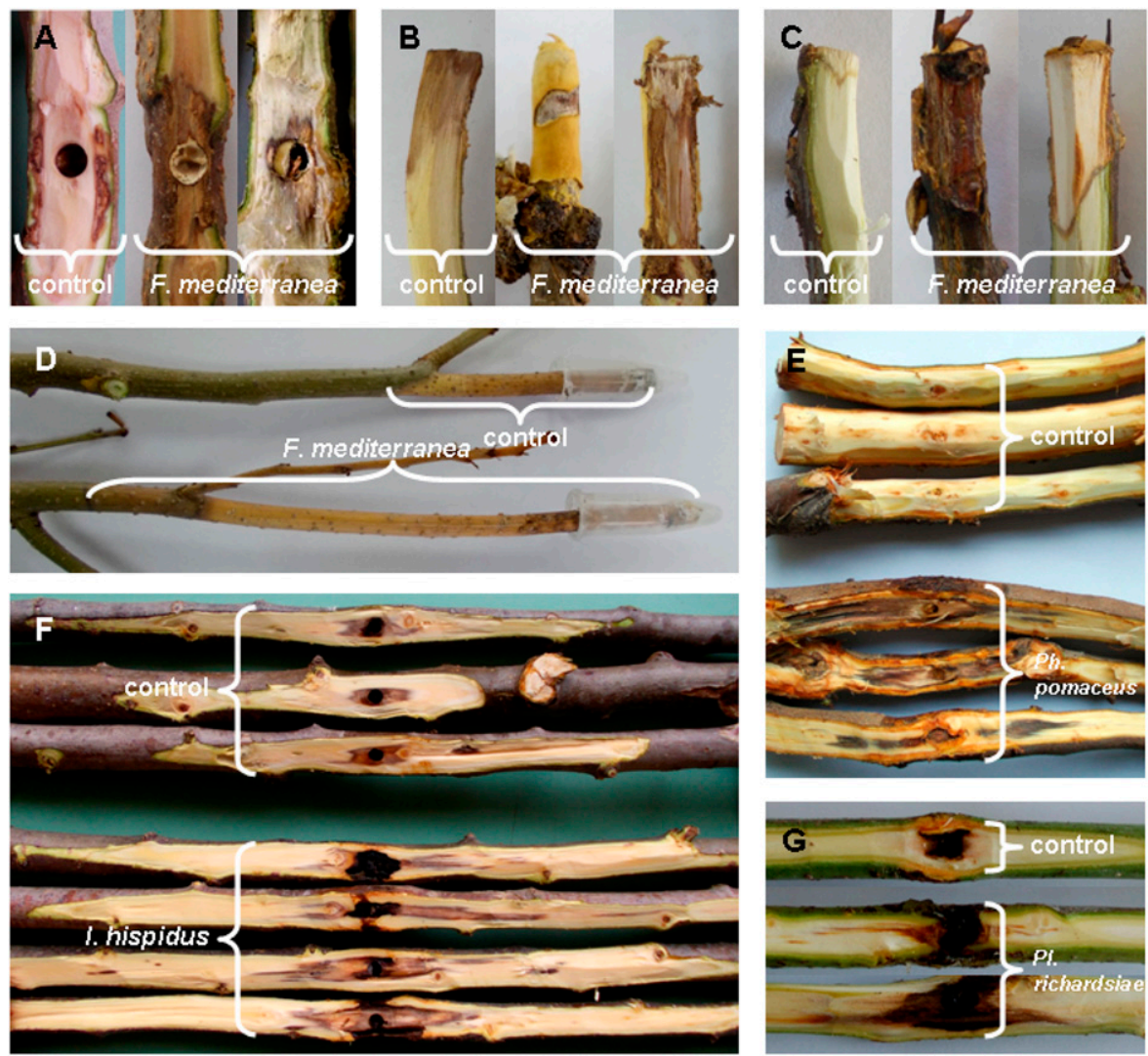

Fig. 4. Wood discoloration and decay symptoms in olive tree $\mathbf{A}$, pomegranate tree $\mathbf{B}$, kumquat tree $\mathbf{C}$, and silk tree $\mathbf{D}$, artificially infested with Fomitiporia mediterranea, and in almond tree $\mathbf{E}$, apple tree $\mathbf{F}$, and avocado tree $\mathbf{G}$, artificially infested with Phellinus pomaceus, Inonotus hispidus, and Pleurostomophora richardsiae, respectively. 
are laborious and time-consuming. In some cases, no significant differences in disease development between control and artificially inoculated plants were observed, even after a long incubation period (Di Marco et al. 2004; Laveau et al. 2009; Paplomatas et al. 2006a). In other cases, the observed differences were marginally significant (Roccotelli et al. 2014), suggesting the extremely slow progress in disease development. In the present study, by employing the trunk-drilling method, all plant species inoculated with $F$. mediterranea strains that were isolated from

Table 4. Pathogenicity of fungal isolates on their hosts and cross-pathogenicity of the F. mediterranea isolate A2LKP1 from grapevine on olive, pomegranate, kumquat, apple, and almond trees

\begin{tabular}{|c|c|c|c|c|c|c|}
\hline \multirow[t]{2}{*}{ Host inoculated } & \multirow[t]{2}{*}{ Fungal species } & \multirow[t]{2}{*}{ Isolate } & \multirow[t]{2}{*}{ Host of origin } & \multicolumn{2}{|c|}{$\begin{array}{l}\text { Discoloration length from the } \\
\text { inoculation point }(\mathrm{mm})^{\mathrm{y}}\end{array}$} & \multirow[t]{2}{*}{ Reisolation $^{\mathrm{z}}$} \\
\hline & & & & Above & Below & \\
\hline \multirow[t]{3}{*}{ Grapevine } & Control & & & $5.1 \pm 0.9 \mathrm{a}$ & $6.1 \pm 1.0 \mathrm{a}$ & - \\
\hline & F. mediterranea & A2LKP1 & Grapevine & $42.0 \pm 4.2 \mathrm{~b}$ & $52.3 \pm 5.1 \mathrm{~b}$ & + \\
\hline & Ph. chlamydospora & $\mathrm{A} 2 \mathrm{LP} 1$ & Grapevine & $79.6 \pm 7.6 \mathrm{c}$ & $90.2 \pm 11.4 \mathrm{c}$ & + \\
\hline \multirow[t]{4}{*}{ Olive } & Control & & & $4.9 \pm 0.2 \mathrm{a}$ & $5.1 \pm 0.6 \mathrm{a}$ & - \\
\hline & F. mediterranea & E3LK1 & Olive tree & $23.2 \pm 2.6 \mathrm{~b}$ & $21.6 \pm 3.4 \mathrm{~b}$ & + \\
\hline & Pl. richardsiae & E1PK1 & Olive tree & $16.5 \pm 2.3 \mathrm{~b}$ & $16.5 \pm 2.3 b$ & + \\
\hline & F. mediterranea & A2LKP1 & Grapevine & $18.7 \pm 1.3 \mathrm{~b}$ & $17.9 \pm 2.4 \mathrm{~b}$ & + \\
\hline \multirow[t]{3}{*}{ Pomegranate } & Control & & & $5.7 \pm 0.2 \mathrm{a}$ & $6.5 \pm 0.3 \mathrm{a}$ & - \\
\hline & F. mediterranea & R1LK1 & Pomegranate tree & $13.0 \pm 1.4 \mathrm{~b}$ & $15.9 \pm 2.7 \mathrm{~b}$ & + \\
\hline & F. mediterranea & A2LKP1 & Grapevine & $7.1 \pm 0.9 \mathrm{a}$ & $6.0 \pm 1.1 \mathrm{a}$ & - \\
\hline \multirow[t]{3}{*}{ Kumquat } & Control & & & $2.5 \pm 0.2 \mathrm{a}$ & $2.6 \pm 0.3 \mathrm{a}$ & - \\
\hline & F. mediterranea & K1KP1 & Kumquat tree & $8.4 \pm 1.5 b$ & $8.9 \pm 1.7 \mathrm{~b}$ & + \\
\hline & F. mediterranea & A2LKP1 & Grapevine & $2.7 \pm 0.2 \mathrm{a}$ & $2.8 \pm 0.2 \mathrm{a}$ & - \\
\hline \multirow[t]{2}{*}{ Pear } & Control & & & $3.2 \pm 0.1 \mathrm{a}$ & $5.7 \pm 0.7 \mathrm{a}$ & - \\
\hline & F. mediterranea & AXL1LK1 & Pear tree & $23.3 \pm 3.7 \mathrm{~b}$ & $19.6 \pm 2.3 \mathrm{~b}$ & + \\
\hline \multirow[t]{3}{*}{ Apple } & Control & & & $14.0 \pm 0.6 \mathrm{a}$ & $12.8 \pm 0.8 \mathrm{a}$ & - \\
\hline & I. hispidus & MP1 & Apple tree & $72.6 \pm 13.5 b$ & $55.3 \pm 9.1 \mathrm{~b}$ & + \\
\hline & F. mediterranea & A2LKP1 & Grapevine & $20.5 \pm 1.9 \mathrm{a}$ & $16.4 \pm 1.4 \mathrm{a}$ & - \\
\hline \multirow[t]{3}{*}{ Almond } & Control & & & $3.2 \pm 0.2 \mathrm{a}$ & $2.7 \pm 0.1 \mathrm{a}$ & - \\
\hline & Ph. pomaceus & AM2XL1 & Almond tree & $47.1 \pm 8.3 \mathrm{~b}$ & $50.0 \pm 6.3 \mathrm{~b}$ & + \\
\hline & F. mediterranea & A2LKP1 & Grapevine & $5.0 \pm 1.1 \mathrm{a}$ & $7.1 \pm 1.2 \mathrm{a}$ & - \\
\hline \multirow[t]{2}{*}{ Avocado } & Control & & & $9.3 \pm 0.9 \mathrm{a}$ & $13.0 \pm 1.7 \mathrm{a}$ & - \\
\hline & Pl. richardsiae & AV1LK3 & Avocado tree & $63.9 \pm 9.6 \mathrm{~b}$ & $49.0 \pm 6.8 \mathrm{~b}$ & + \\
\hline \multirow[t]{2}{*}{ Mulberry } & Control & & & $24.0 \pm 1.0 \mathrm{a}$ & $22.7 \pm 0.9 \mathrm{a}$ & - \\
\hline & F. solani & MOYR2P3 & Mulberry tree & $74.7 \pm 1.9 \mathrm{~b}$ & $83.9 \pm 10.0 \mathrm{~b}$ & + \\
\hline
\end{tabular}

y The pathogenicity of Fomitiporia mediterranea, Phaeomoniella chlamydospora, Pleurostomophora richardsiae, Inonotus hispidus, Phellinus pomaceus and Fusarium solani isolates was evaluated on the basis of the extension of wood tissue discoloration above and below the inoculation point after a 7-month incubation period. Artificial inoculations were conducted by employing the trunk-drilling method and inserting a 5.5-mm-diameter mycelial disc taken from the margin of a 15-day-old PDA culture. Control plants were mock-inoculated with a 5.5-mm-diameter sterilized PDA plug. According to Tukey's HSD test at $P<0.05$, values followed by the same letter did not differ significantly within each plant species inoculated.

${ }^{\mathrm{z}}$ Pathogen reisolation was performed by making longitudinal sections of the trunk and placing 10 wood chips taken from the symptomatic wood, above and below the inoculation point, onto acidified PDA. Plates were incubated at $24^{\circ} \mathrm{C}$ in the dark for 3 weeks. In each plant-pathogen pathosystem, at least 3 plants were used for pathogen reisolation. Symbols ' + ' and '-' represent successful and unsuccessful pathogen reisolation, respectively.

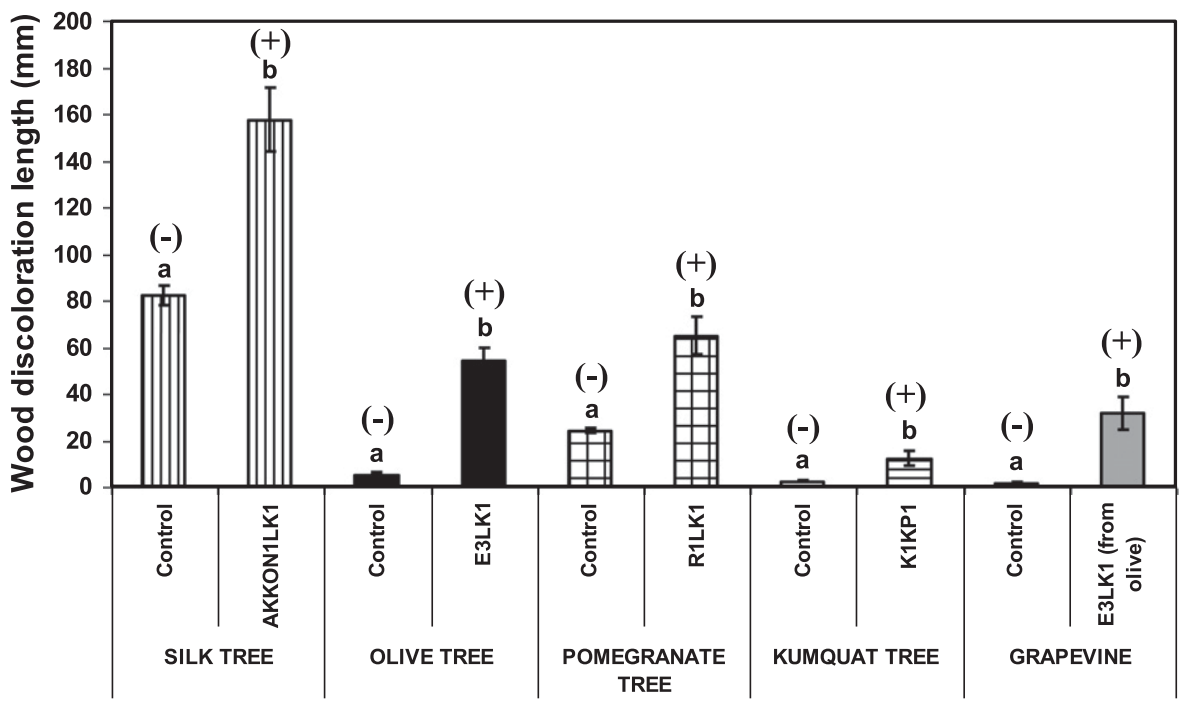

Fig. 5. Length of wood tissue discoloration below the inoculation point of silk, olive, pomegranate, kumquat, and grapevine stems or branches, 7 months after adjusting mycelial discs of Fomitiporia mediterranea isolates onto fresh pruning wounds. Control plants were mock inoculated with sterilized PDA discs. Within each plant species, columns followed by different letters differ significantly, according to the $t$ test at $P<0.001$. Vertical bars indicate standard errors. Above each column, symbols ' + ' and '-' into brackets represent successful and unsuccessful pathogen reisolation, respectively. 
the same host, exhibited significantly higher disease reaction compared with their mock-inoculated controls. Moreover, the fungus was successfully reisolated from diseased tissues, but not from controls, thus confirming its pathogenicity. However, the extension of wood discoloration was much lower in pomegranate and kumquat trees than in other hosts inoculated. Given that $F$. mediterranea penetrates through wounds (Elena et al. 2006; Mugnai et al. 1999; Roccotelli et al. 2014), we attempted to simulate the natural infection process and prompt disease development by using the pruning-wound method to inoculate grapevine, olive, pomegranate, kumquat, and silk tree. The pruningwound inoculation approach was proved to be a robust method since most inoculated plants exhibited typical wood decay symptoms, similar to those observed under natural infection conditions. Moreover, the extension of wood tissue lesions in olive and pomegranate was greater compared with those observed in trunk-drilling inoculated plants, whereas lesions' length in kumquat remained low. Other researchers have likewise employed the pruning-wound instead of the trunkdrilling inoculation method to evaluate pathogenicity (Gonzalez et al. 2009; Sparapano et al. 2000). In addition, other citrus species have also shown a very narrow disease reaction in pathogenicity tests with $F$. mediterranea (Elena et al. 2006; Roccotelli et al. 2014).

It has often been documented that $F$. mediterranea isolates vary in morphology and physiology onto solid culture media (Fischer 2002; 2006; Pilotti et al. 2005; 2010; Roccotelli et al. 2014). Therefore, Fischer (1987) divided $F$. mediterranea isolates into either bleaching (B) or staining (S) type: in type B, the mycelium in solid media is cottony to woolly, forming yellow to brown hyphae, and exhibiting weak or no pigmentation of the medium and fast growth rates; whereas in type $\mathrm{S}$, the mycelium is characterized by sparse aerial hyphae, stronger pigmentation of the medium, and slower growth rate. Indeed, F. mediterranea isolates analyzed in the present study differed significantly in growth rate, culture pigmentation on the under- and upper- side, and mycelium abundance. However, classification system proposed by Fischer (1987) was not completely feasible for the $F$. mediterranea isolates examined here. Several isolates exhibited features that were contradictory, thus they could not be placed into either B- or S- type. For instance, isolate E5LKE exhibited the lowest growth rate and intense pigmentation on both sides of cultures, suggesting type $\mathrm{S}$, but also woolly mycelium, indicating type B. In addition, isolate E6LK1 showed the lowest pigmentation on the undersides of cultures, indicating type B; however, its very sparse aerial hyphae indicated type $S$. Therefore, in the present study, classification of isolates into either B- or S- type was carried out by assessing the appearance of pigment on the underside of culture plates, solely, according to Fischer (2006): isolates exhibiting modest to strong pigmentation of the culture medium were designated as type $\mathrm{S}$, whereas those with weak or no pigmentation, as type $\mathrm{B}$. Hence, analysis of cultures' morphology revealed the presence of both $\mathrm{B}$ and $\mathrm{S}$ types within $21 \mathrm{~F}$. mediterranea isolates tested (including the reference strain K.E. Fomit 4), where most of them were type B, as has been reported in other studies (Fischer 2006; Roccotelli et al. 2014). On the other hand, the growth rates and phylogenetic position of the isolates E3LK1, E5LK2, and A2LKP1 are distinct to other F. mediterranea isolates, indicating intraspecies variation possibly linked to the severity of disease symptoms caused by $F$. mediterranea.

Besides $F$. mediterranea, several other important phytopathogenic species such as $I$. hispidus, $P$. pomaceus, $P$. richardsiae, and F. solani are presented for the first time worldwide as the causal agents of wood infection in apple, almond, avocado, and mulberry trees, respectively. Although I. hispidus commonly occurs on ash, apple, and walnut in Europe (Fischer and Gonzalez-Garcia 2015; Schwarze et al. 2000), this is the first experimental evidence of I. hispidus on apple trees by means of pathogenicity tests. Likewise, Gonzalez et al. (2009) reported the presence of I. hispidus carpophores on esca-infected grapevines in Spain, revealing also the pathogenicity of this fungus on healthy vines by in vitro bioassays. Moreover, although $P$. pomaceus is considered as a common plant pathogen of various Prunus species (Lonsdale 1999; Rayner and Boddy 1988), it has never been reported as primary pathogen on almond trees. In addition, $P$. richardsiae has been associated with wood streaking of olive and almond trees in Italy and Spain (Carlucci et al. 2013;
Olmo et al. 2015) and with grapevine trunk diseases in Italy, California, and South Africa (Carlucci et al. 2015; Eskalen et al. 2004; Rolshausen et al. 2010; White et al. 2011). However, this is the first record of $P$. richardsiae as a wood-infecting microorganism in avocado, while the same fungus is associated with wood streaking of olive trees for the first time in Greece. The species $F$. solani is a well-described cosmopolitan plant pathogen, which includes endophytic strains that can act as tissue decomposers (Schroers et al. 2017), although this is the first report of $F$. solani as a wood deterioration inducer in mulberry trees.

Despite the lack of pathogenicity tests, Cosmospora from apple tree, Auricularia aurica-judae (Bull.) J.Schröt. and Aphanobasidiumlike fungi from walnut tree, Phialophora from almond tree, Fuscoporia from olive tree, Didymosphaeria from kumquat tree and grapevine, Phialemonium and Coniophoraceae from kumquat tree, Eutypa from grapefruit tree, Inonotus rickii (Pat.) D.A. Reid from mulberry tree, Perenniporia from Jerusalem sage, Xenoacremonium from pomegranate and chinaberry tree, Trametes from loquat, and T. ljubarskyi Pilát from plane tree were also isolated for the first time from diseased hosts. In addition, several other fungi-namely, the well-described wood decay pathogens Inonotus hispisdus on walnut and mulberry (Schwarze et al. 2000) and Phellinus rimosus (Berk.) Pilát on black locust tree (Riffle et al. 1985)-were detected in the present study for the first time in Greece. However, no pathogenicity tests were conducted for the abovementioned pathosytems in the present study, thus the phytopathogenic potential of these fungi on their hosts remains uncertain.

Moreover, certain basidiomycetes from the present study, which were associated with wood decay of various tree species, were distantly related to their known taxonomic relatives, representing novel phylogenetic linkages among the class Agaricomycetes (Table 2). In particular, isolate KAR1LKON1 possessed a distinct phylogenetic position within the order Agaricales, showing only $82.9 \%$ similarity to an Aphanobasidium sp. Apart from the high similarity to its nontaxonomically described strain $\mathrm{C} 1$, isolate K1AL1 showed 88.7 to $89.1 \%$ phylogenetic relatedness to known Coniophora species, indicating that this strain is a new member of the family Coniophoraceae. Moreover, isolate ROMP1 mLP1 obtained from black locust tree was distantly related to Phellinus/Fulvifomes spp., therefore being a novel Phellinus-like representative.

In view of all the above, wood decay diseases in tree species could be attributed to diverse fungal pathogens. Identification of wood-decay fungi and investigation of their phytopathogenic role is essential to provide successful disease diagnosis and management. Moreover, the further extension of $F$. mediterranea host range along with the potential efficiency of the fungus to transinfect hosts other than its original ones in practice should be taken into account to estimate disease risk and plan future control strategies.

\section{Acknowledgments}

This research work was funded under the Research \& Technology Development Innovation Projects-AgroETAK, MIS 453350 action, in the framework of the Operational Program Human Resources Development, through a post-doctoral grant to Emmanouil A. Markakis. The project was co-funded by the European Social Fund through the National Strategic Reference Framework (Research Funding Program 2007-2013) coordinated by the Hellenic Agricultural Organization DEMETER (Institute of Olive Tree, Subtropical Crops and Viticulture, Chania, Crete, Greece). It was also accomplished with the contribution of the LIFE+ financial instrument of the European Union to project LIFE11 ENV/GR/942 OLIVECLIMA.

\section{Literature Cited}

Bigelow, D. M., Gilbertson, R. L., and Matheron, M. E. 1998. Cultural studies of fungi causing brown rot in heartwood of living lemon trees in Arizona. Mycol. Res. 102:257-262.

Bigelow, D. M., Matheron, M. E., and Gilbertson, R. L. 1996. Biology and control of Coniophora eremophila on lemon trees in Arizona. Plant Dis. 80:934-939.

Binder, M., Larsson, K.-H., Matheny, P. B., and Hibbett, D. S. 2010. Amylocorticiales ord. nov. and Jaapiales ord. nov.: Early diverging clades of Agaricomycetidae dominated by corticioid forms. Mycologia 102:865-880.

Blanchette, R. A. 1991. Delignification by wood-decay fungi. Annu. Rev. Phytopathol. 29:381-398.

Brazee, N. J. 2015. Phylogenetic relationships among species of Phellinus sensu stricto, cause of white trunk rot of hardwoods, from northern North America. Forests 6:4191-4211. 
Butin, H. 1995. Tree Diseases and Disorders. Oxford University Press, Oxford

Carlucci, A., Cibelli, F., Lops, F., Phillips, A. J. L., Ciccarone, C., and Raimondo, M. L. 2015. Pleurostomophora richardsiae associated with trunk diseases of grapevine in southern Italy. Phytopathol. Mediterr. 54:109-123.

Carlucci, A., Raimondo, M. L., Cibelli, F., Phillips, A. J. L., and Lops, F. 2013. Pleurostomophora richardsiae, Neofusicoccum parvum and Phaeoacremonium aleophilum associated with a decline of olives in southern Italy. Phytopathol. Mediterr. 52:517-527.

Dai, Y. C., D'Amico, L., Motta, E., and Annesi, T. 2010. First report of Inonotus rickii causing canker and decay on Hevea brasiliensis in China. Plant Pathol. 59:806.

Damm, U., Verkley, G. J. M., Crous, P. W., Fourie, P. H., Haegi, A., and Riccioni, L. 2008. Novel Paraconiothyrium species on stone fruit trees and other woody hosts. Persoonia 20:9-17.

Di Marco, S., Calzarano, F., Osti, F., and Mazzullo, A. 2004. Pathogenicity of fungi associated with a decay of kiwifruit. Austr. Plant Pathol. 33:337-342.

Elena, K., Fischer, M., Dimou, K., and Dimou, D. M. 2006. Fomitiporia mediterranea infeting citrus trees in Greece. Phytopathol. Mediterr. 45:35-39.

Elena, K., and Paplomatas, E. J. 2002. First report of Fomitiporia punctata infecting kiwifruit. Plant Dis. 86:1176.

Eskalen, A., Latham, S. R., and Gubler, W. D. 2004. Pathogenicity of Phialophora sp. on grapevines in California. Phytopathol. Mediterr. 43:147.

Fischer, M. 1987. Biosystematische Untersuchungen an den Porlingsgattungen Phellinus Quél. und Inonotus Karst. Bibl. Mycol. 107:1-133.

Fischer, M. 2002. A new wood-decaying basidiomycete species associated with esca of grapevine: Fomitiporia mediterranea (Hymenochaetales). Mycol. Prog. 1:315-324.

Fischer, M. 2006. Biodiversity and geographic distribution of basidiomycetes causing esca-associated white rot in grapevine: a worldwide perspective. Phytopathol. Mediterr. 45:30-42.

Fischer, M., and Gonzalez-Garcia, V. 2015. An annotated checklist of European basidiomycetes related to white rot of grapevine (Vitis vinifera). Phytopathol. Mediterr. 54:281-298.

Gonzalez, V., Sanchez-Torrez, P., Hinarejos, R., and Tuset, J. J. 2009. Inonotus hispidus fruiting bodies on grapevines with esca symptoms in Mediterranean areas of Spain. J. Plant Pathol. 91:465-468

Graham, G. H., Brlansky, R. H., Timmer, L. W., Lee, R. F., Marais, L. G., and Bender, G. S. 1985. Comparison of citrus tree declines with necrosis of major roots and their association with Fusarium solani. Plant Dis. 69:1055-1058.

Groenewald, M., Kang, J. C., Crous, P. W., and Gams, W. 2001. ITS and beta-tubulin phylogeny of Phaeoacremonium and Phaeomoniella. Mycol. Res. 105:651-657.

Grünig, C. R., Sieber, T. N., Rogers, S. O., and Holdenrieder, O. 2002. Genetic variability among strains of Phialocephala fortinii and phylogenetic analysis of the genus Phialocephala based on rDNA ITS sequence comparisons. Can. J. Bot. 80:1239-1249.

Guglielmo, F., Bergemann, S. E., Gonthier, P., Nicolotti, G., and Garbelotto, M. 2007. A multiplex PCR-based method for the detection and early identification of wood rotting fungi in standing trees. J. Appl. Microbiol. 103:1490-1507.

Herrera, C. S., Rossman, A. Y., Samuels, G. J., and Chaverri, P. 2013. Pseudocosmospora - a new genus to accommodate Cosmospora vilior and related species. Mycologia 105:1287-1305.

Huang, X., and Madan, A. 1999. CAP3: A DNA sequence assembly program. Genome Res. 9:868-877.

Johnston, P. R., Park, D., and Manning, M. A. 2010. Neobulgaria alba sp. nov. and its Phialophora-like anamorph in native forests and kiwifruit orchards in New Zealand. Mycotaxon 113:385-396.

Laveau, C., Letouze, A., Louvet, G., Bastien, S., and Guerin-Dobrana, L. 2009. Differential aggressiveness of fungi implicated in esca and associated diseases of grapevine in France. Phytopathol. Mediterr. 48:32-46.

Lombard, L., van der Merwe, N. A., Groenewald, J. Z., and Crous, P. W. 2015. Generic concepts in Nectriaceae. Stud. Mycol. 80:189-245.

Lonsdale, D. 1999. Research for Amenity Trees. The Stationery Office, London.

Mayorquin, J. S., Wang, D. H., Twizeyimana, M., and Eskalen, E. 2016. Identification, distribution and pathogenicity of Ditrypaceae and Botryosphaeriaceae associated with citrus branch canker in the southern California desert. Plant Dis. 100: 2402-2413.

Mugnai, L., Graniti, A., and Surico, G. 1999. Esca (black measles) and brown-wood streaking: two old and elusive diseases of grapevines. Plant Dis. 83:404-418.

Olmo, D., Armengol, J., Leon, M., and Gramaje, D. 2015. Pathogenicity testing of lesser-known fungal trunk pathogens associated with wood decay of almond trees. Eur. J. Plant Pathol. 143:607-611.

Paplomatas, E. J., Elena, K., Tsopelas, P., Tzima, A., Paraskevopoulos, A., and Papanikolaou, A. 2006a. Infection of olive trees with the fungus Fomitiporia punctata (Phellinus punctatus). Phytopathol. Mediterr. 45:69.
Paplomatas, E. J., Paraskevopoulos, A., Tsopelas, P., Elena, K., and Malandraki, I. 2006b. Study of the epidemiology of esca disease on olive. Phytopathol. Mediterr. 45:166-167.

Pilotti, M., Gervasi, F., and Brunetti, A. 2005. Molecular identification of Fomitiporia mediterranea and Eutypa lata/Libertella blepharis in Platanus $\times$ acerifolia. J. Phytopathol. 153:193-202.

Pilotti, M., Tizzani, L., Brunetti, A., Gervasi, F., Di Lernia, G., and Lumia, V. 2010. Molecular identification of Fomitiporia mediterranea on declining and decayed hazelnut. J. Plant Pathol. 92:115-129.

Rayner, A. D. M., and Boddy, L. 1988. Fungal Decomposition of Wood: Its Biology and Ecology. Wiley Interscience, Chichester, UK

Riffle, J. W., Myatt, A. K., and Davis, R. L. 1985. Incidence of Phellinus robineae in black locust plantings in Oklahoma. Plant Dis. 69:116-118.

Roccotelli, A., Schena, L., Sanzani, S. M., Cacciola, S. O., Mosca, S., Faedda, R., Ippolito, A., and Magnano di San Lio, G. 2014. Characterization of basidiomycetes associated with wood rot of citrus in southern Italy. Phytopathology 104:851-858.

Rolshausen, P. E., Urbez-Torres, J. R., Rooney-Latham, S., Eskalen, A., Smith, R. J., and Gubler, W. D. 2010. Evaluation of pruning wound susceptibility and protection against fungi associated with grapevine trunk diseases. Am. J. Enol. Vitic. 61: 113-119.

Saitou, N., and Nei, M. 1987. The neighbor-joining method: A new method. for reconstructing phylogenetic trees. Mol. Biol. Evol. 4:406-425.

Schmidt, O., Gaiser, O., and Dujesiefken, D. 2012. Molecular identification of decay fungi in the wood of urban trees. Eur. J. For. Res. 131:885-891.

Schmidt, O., Grimm, K., and Moreth, U. 2002. Molecular identity of species and isolates of the Coniophora cellar fungi. Holzforschung 56:563-571.

Schroers, H. J., Samuels, G. J., Zhang, N., Short, D. P., Juba, G. H., and Geiser, D. M 2017. Epitypification of Fusisporium (Fusarium) solani and its assignment to a common phylogenetic species in the Fusarium solani species complex. Mycologia 108:806-819.

Schwarze, F. W. M. R., Engels, J., and Mattheck, C. 2000. Fungal Strategies of Wood Decay in Trees, 1 st Ed. Springer, Berlin.

Sievers, F., Wilm, A., Dineen, D., Gibson, T. J., Karplus, K., Li, W., Lopez, R. McWilliam, H., Remmert, M., Söding, J., Thompson, J. D., and Higgins, D. G. 2011. Fast, scalable generation of high-quality. protein multiple sequence alignments using Clustal Omega. Mol. Syst. Biol. 7:539.

Sparapano, L., Bruno, G., Ciccarone, C., and Graniti, A. 2000. Infection of grapevines by some fungi associated with esca. Fomitiporia punctata as a wood-rot inducer. Phytopathol. Mediterr. 39:46-52.

Surico, G., Mugnai, L., and Marchi, G. 2006. Older and more recent observations on esca: A critical overview. Phytopathol. Mediterr. 45:68-86.

Surico, G., Mugnai, L., and Marchi, G. 2008. The esca disease complex. Pages 119-136 in: Integrated Management of Diseases Caused by Fungi, Phytoplasma and Bacteria. Ciancio, A., and Mukerji, K., eds. Springer, Dordrecht.

Tamura, K., and Nei, M. 1993. Estimation of the number of nucleotide. substitutions in the control region of mitochondrial DNA in humans and. chimpanzees. Mol. Biol. Evol. 10:512-526.

Tamura, K., Stecher, G., Peterson, D., Filipski, A., and Kumar, S. 2013. MEGA6 Molecular evolutionary genetics analysis version 6.0. Mol. Biol. Evol. 30:2725-2729.

Thorn, R. J., Reddy, C. A., Harris, D., and Paul, E. A. 1996. Isolation of saprophytic basidiomycetes from soil. Appl. Environ. Microbiol. 62:4288-4292.

Tomšovský, M., Kolaří, M., Pažoutová, S., and Homolka, L. 2006. Molecular phylogeny of European Trametes (Basidiomycetes, Polyporales) species based on LSU and ITS (nrDNA) sequences. Nova Hedwigia 82:269-280.

Trouillas, F. P., and Gubler, W. D. 2004. Identification and characterization of Eutypa leptoplaca, a new pathogen of grapevine in Northern Califonria Mycol. Res. 108:1195-1204.

Úrbez-Torres, J. R., Peduto, F., Vossen, P. M., Krueger, W. H., and Gubler, W. D. 2013. Olive twig and branch dieback: Etiology, incidence, and distribution in California. Plant Dis. 97:231-244.

White, C. L., Halleen, F., and Mostert, L. 2011. Symptoms and fungi associate with esca in South African vineyards. Phytopathol. Mediterr. 50:236-246.

White, T. J., Bruns, T., Lee, S., and Taylor, J. 1990. Amplification and direct sequencing of fungal ribosomal RNA genes for phylogenies. Pages 315-322 in: PCR Protocols. A Guide to Methods and Applications. M. A. Innis, D. H. Gelfand, J. J. Sninsky, and J. W. White, eds. Academic Press, New York.

Zhao, C. L., and Cui, B. K. 2012. A new species of Perenniporia (Polyporales, Basidiomycota) described from southern China based on morphological and molecular characters. Mycol. Prog. 11:555-560.

Zhao, C. L., and Cui, B. K. 2013. Morphological and molecular identification of four new resupinate species of Perenniporia (Polyporales) from southern China. Mycologia 105:945-958. 